International Journal of Biological Sciences ISSN 1449-2288 www.biolsci.org 2005 1:51-66

Review

Received: 2004.07.23 Accepted: 2005.02.01 Published: 2005.04.01

Abstract

Author biography

\section{The GAPs, GEFs, and GDls of heterotrimeric G-protein alpha subunits}

\section{David P. Siderovski and Francis S. Willard}

Department of Pharmacology, UNC Lineberger Comprehensive Cancer Center, and UNC Neuroscience Center, The University of North Carolina at Chapel Hill, CB\#7365, 1106 M.E. Jones Building, Chapel Hill, NC 27599-7365 USA

The heterotrimeric G-protein alpha subunit has long been considered a bimodal, GTP-hydrolyzing switch controlling the duration of signal transduction by seven-transmembrane domain ( $7 \mathrm{TM}$ ) cell-surface receptors. In 1996, we and others identified a superfamily of "regulator of G-protein signaling" (RGS) proteins that accelerate the rate of GTP hydrolysis by G $\alpha$ subunits (dubbed GTPase-accelerating protein or "GAP" activity). This discovery resolved the paradox between the rapid physiological timing seen for 7TM receptor signal transduction in vivo and the slow rates of GTP hydrolysis exhibited by purified $\mathrm{G} \alpha$ subunits in vitro. Here, we review more recent discoveries that have highlighted newly-appreciated roles for RGS proteins beyond mere negative regulators of 7TM signaling. These new roles include the RGS-box-containing, RhoA-specific guanine nucleotide exchange

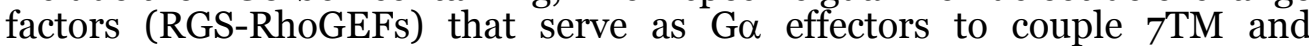
semaphorin receptor signaling to RhoA activation, the potential for RGS12 to serve as a nexus for signaling from tyrosine kinases and G-proteins of both the $\mathrm{G} \alpha$ and Ras-superfamilies, the potential for R7-subfamily RGS proteins to couple $\mathrm{G} \alpha$ subunits to $7 \mathrm{TM}$ receptors in the absence of conventional G $\beta \gamma$ dimers, and the potential for the conjoint 7TM/RGS-box Arabidopsis protein AtRGS1 to serve as a ligand-operated GAP for the plant G $\alpha$ AtGPA1. Moreover, we review the discovery of novel biochemical activities that also impinge on the guanine nucleotide binding and hydrolysis cycle of $\mathrm{G} \alpha$ subunits: namely, the guanine nucleotide dissociation inhibitor (GDI) activity of the GoLoco motif-containing proteins and the 7TM receptorindependent guanine nucleotide exchange factor (GEF) activity of Ric-8/synembryn. Discovery of these novel GAP, GDI, and GEF activities have helped to illuminate a new role for Ga subunit GDP/GTP cycling required for microtubule force generation and mitotic spindle function in chromosomal segregation.

asymmetric cell division, GoLoco motif, G-protein, RGS proteins, RIC-8

David P. Siderovski received his Ph.D. from the University of Toronto and is currently an Associate Professor of Pharmacology at the University of North Carolina at Chapel Hill. Dr. Siderovski was one of the first scientists to identify the regulators of G-protein signaling (RGS) protein superfamily. In April 2004, Dr. Siderovski received the John J. Abel Award from the American Society for Pharmacology and Experimental Therapeutics for his contributions that have helped shape the field of pharmacology and challenged previously established dogmas in the field of G protein-coupled receptor signaling. Dr. Siderovski's current work focuses on elucidating the specific roles of RGS proteins as scaffolds in the coordination of multiple signal transduction pathways, as well as in modulation of microtubule dynamics at the mitotic spindle in dividing cells.

Francis S. Willard obtained a Bachelor's of Science with first-class honours in Physiology at Victoria University, Wellington, New Zealand. He went on to complete his Ph.D. at the Australian National University in 2002, and in 2003 received the prestigious Frank Fenner Medal for his doctoral work on growth factor signal transduction pathways. Dr. Willard is currently working with Dr. Siderovski as a senior postdoctoral fellow in the UNC Department of Pharmacology and is utilizing biochemical and structural approaches to understand the role of G-protein signaling in developmental biology.

David P. Siderovski, dsiderov@med.unc.edu tel: 1-919-843-9363 fax: 1-919-966-5640 


\section{Introduction}

Heterotrimeric guanine-nucleotide binding proteins (G-proteins) are best known for transducing a wide array of extracellular signals received from heptahelical (7TM) cell-surface receptors, such as those initiated by hormones in the bloodstream, neurotransmitters across the synapse, and photons striking the retina. In the conventional model of heterotrimeric G-protein activation, the ligand/7TM receptor interaction catalyzes guanine nucleotide exchange on the G $\beta \gamma$-complexed (and GDP-bound) G $\alpha$ subunit (Fig. 1). The G $\alpha$ GTP and G $\beta \gamma$ subunits of the heterotrimer then dissociate and are thus free to propagate the signal forward via separate (and sometimes converging) interactions with adenylyl cyclases, phospholipase-C isoforms, potassium and calcium ion channels, guanine-nucleotide exchange factors for the GTPase RhoA ("RhoGEFs"), and other "effector" enzymes [1, 2, 3]. The intrinsic GTP hydrolysis (GTPase) activity of the

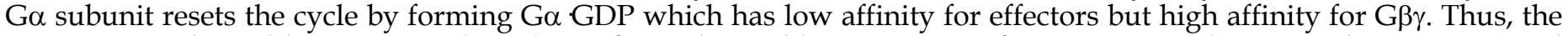
inactive GDP-bound heterotrimer $(\mathrm{G} \alpha \beta \gamma)$ is reformed, capable once again of interacting with activated receptor. Based on this cycle, the duration of heterotrimeric G-protein signaling is thought to be controlled by the lifetime of the Ga subunit in its GTP-bound state. In 1996, we and others identified a superfamily of RGS ("regulator of G-protein signaling") proteins that bind $\mathrm{G} \alpha$ subunits via a hallmark 120 amino-acid "RGS-box" domain [4, 5, 6, 7, 8, 9], dramatically accelerating their intrinsic GTPase activity [10, 11, 12], and thus attenuating heterotrimer-linked signaling (reviewed in $[13,14,15]$ ). The discovery of the RGS proteins and their GTPase-accelerating (or "GAP") activity on Ga subunits not only resolved apparent timing paradoxes between known 7TM-receptor-mediated physiological responses and the activity in vitro of the responsible G-proteins (e.g., [16]), but in addition, novel functional domains discovered within members of the RGS protein superfamily (Fig. 2) have helped to reveal hitherto unappreciated roles for Ga subunits in cellular processes outside the realm of 7TM receptor signaling.

Figure 1. Standard model of the guanine nucleotide cycle governing $7 \mathrm{TM}$ receptor-mediated activation of heterotrimeric G protein-coupled signaling. The $G \beta \gamma$ heterodimer serves to couple $\mathrm{G} \alpha$ to the receptor and also to inhibit its spontaneous release of GDP (i.e., acting as a guanine nucleotide dissociation inhibitor or "GDI" for G $\alpha$ [17, 18]). Ligand-occupied, 7TM cell-surface receptors stimulate signal onset by acting as guanine nucleotide exchange factors (GEFs) for $\mathrm{G} \alpha$ subunits, facilitating GDP release, subsequent binding of GTP, and release of the G $\beta \gamma$ dimer. Both the GTP-bound G $\alpha$ and liberated $\mathrm{G} \beta \gamma$ moieties are then able to modulate the activity of various enzymes, ion channels, and other effectors. Regulator of G-protein signaling (RGS) proteins stimulate signal termination by acting as GTPase-accelerating proteins (GAPs) for $\mathrm{G} \alpha$, dramatically enhancing their intrinsic rate of GTP

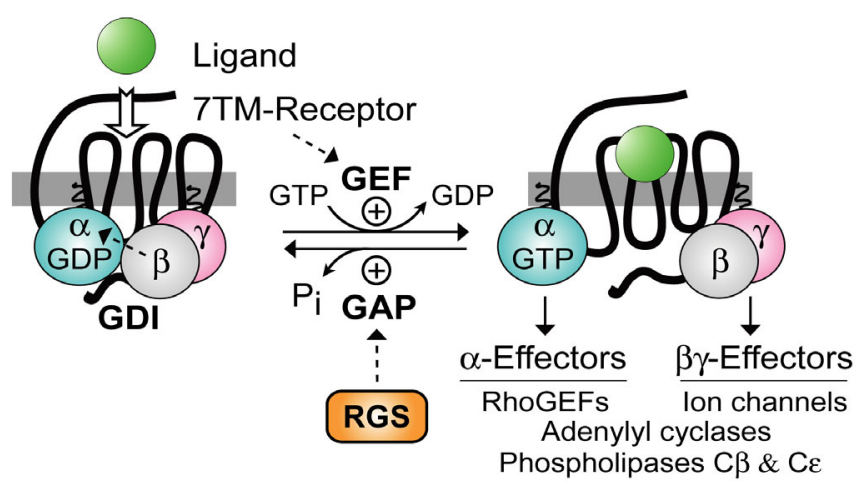
hydrolysis.

\section{The spectrum of RGS protein structure and function}

Founding members of the RGS protein superfamily were discovered in 1996 in a wide spectrum of species: "supersensitivity to pheromone-2" (Sst2) in the budding yeast Saccharomyces cerevisiae [5, 19, 20], FlbA in the aspergillus Emericella nidulans [9], EGL-10 in the nematode worm Caenorhabiditis elegans [7], and RGS1 and RGS2 from human Band T-lymphocytes, respectively [6, 8]. Nearly a decade later, new RGS-box-containing proteins are still being identified in mammalian species (e.g., humans in Fig. 3) and even in plants (Fig. 4C). For example, RGS21 was recently identified as a putative component of taste bud signal transduction that is transacted in lingual epithelium via the T1R2/T1R3 sweet taste and T2R bitter taste 7TM receptors [21]. At a mere 152 amino-acids, RGS21 is the shortest RGS protein known to date, with no obvious structural domain N- or C-terminal to the central RGS-box. The singular nature of the RGS21 domain structure is typical of the A/RZ and B/R4 subfamilies (Figs. 2\&3) but is unlike other superfamily members that either contain two or more RGS-boxes in tandem (i.e., the PKA regulatory subunit binding partner D-AKAP2 [22] and the testes-specific protein PRTD-NY2 [a.k.a. RGS22; Willard \& Siderovski, unpublished observations]) or possess one or more functional modules beyond the defining RGS-box (Fig. 2). Several recent findings as to the functions of these multi-domain RGS proteins are described below.

Figure 2. Multidomain architecture of representative members from all subfamilies of the mammalian RGS protein superfamily. Two alternative nomenclatures have been proposed for several RGS protein subfamilies [23, 24]. RZ- or A-subfamily members such as RGS17 [25] are characterized by an N-terminal poly-cysteine region ("Cys") thought to be reversibly palmitoylated [26]. R4- or B-subfamily members include RGS2 and RGS21 that are described in the text. RGS11 was the first member of the R7- or C-subfamily to be shown to bind G $\beta 5$ via its G $\gamma$-like or "GGL" domain [27]. Of the three members of the R12- or D-subfamily (RGS10, RGS12, RGS14), RGS10 is the smallest and comprises little more than an RGS-box [11], whereas both RGS12 and RGS14 have tandem Ras-binding domains (RBDs) and a C-terminal Gai/o-Loco interaction (GoLoco) motif [28], and RGS12 additionally has N-terminal PDZ (PSD95/Dlg/ZO-1 homology) and PTB (phosphotyrosine-binding) domains [29]. Axin and Axin2 (a.k.a. Axil) are negative regulators of the Wnt signaling pathway and comprise the RA- or E-subfamily; neither protein has been shown to interact with $\mathrm{G \alpha}$ subunits, but rather interact with the tumor suppressor protein adenomatous polyposis coli (APC) using the RGS-box [30]. Axin and Axil also contain other domains that interact with $\beta$-catenin, the kinase GSK3 $\beta$, the phosphatase PP2A, and the protein Dishevelled ("DIX" domain) [31]. The GEF- or F-subfamily includes three RhoA-specific guanine nucleotide exchange factors ("GEFs") with canonical Dbl-homology (DH) and pleckstrin-homology (PH) domains: p115RhoGEF, PDZ-RhoGEF, and leukemia-associated RhoGEF (LARG); the latter two RhoGEFs each possess an N-terminal PDZ 
domain, as described in the text. In 1996, we were the first group to identify [8] an N-terminal RGS-box within each member of the G protein-coupled receptor kinase family (known as the GRK- or G-subfamily in the context of the RGS protein superfamily). At least three sorting nexins (SNX13, SNX14, SNX25) have RGS-boxes between phosphatidylinositol-binding (PX) and PX-associated (PXA) domains and thus comprise the SNX- or H-subfamily of RGS proteins. Zheng and colleagues reported that SNX13 (a.k.a. "RGS-PX1") could act as a GAP for the adenylyl-cyclase-stimulatory isoform of $\mathrm{G \alpha}\left(\mathrm{G \alpha}_{\mathrm{s}}\right)$ [32]; however, this report has yet to be confirmed in the literature. TM, putative transmembrane regions. The multiple RGS-box family members D-AKAP2 and RGS22 fall outside the eight established subfamilies; the superscript designations of their RGS-boxes match that used in Figure 3.

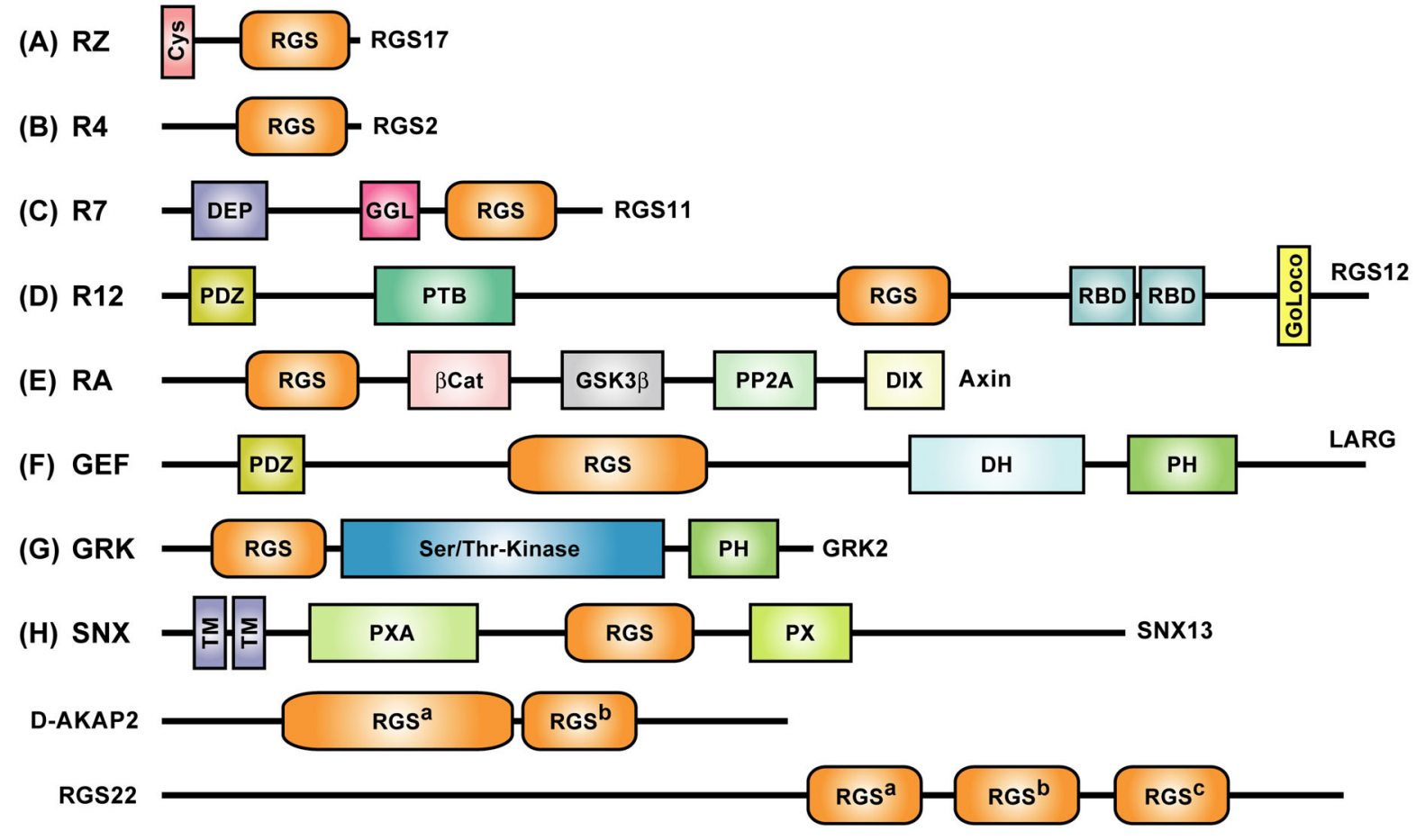

Figure 3. Relationship between RGS-box sequences of all 37 human RGS proteins identified to date. Unrooted dendrogram was generated by Clustal-W [33] and TreeView [34] using sequences identified by the SMART profile [35] for RGSboxes as well as those identified by protein-fold recognition algorithms [36]. Subfamily designations and identification of isolated RGSbox sequences from multiRGS-containing proteins DAKAP2 and RGS22 are as described for Figure 2. Note that there is no RGS15, contrary to an early report [7].

\section{2.a. R7 RGS proteins as novel $\mathbf{G} \gamma$ subunits}

In 1998, we identified a polypeptide sequence, $\mathrm{N}$ terminal to the RGS-box

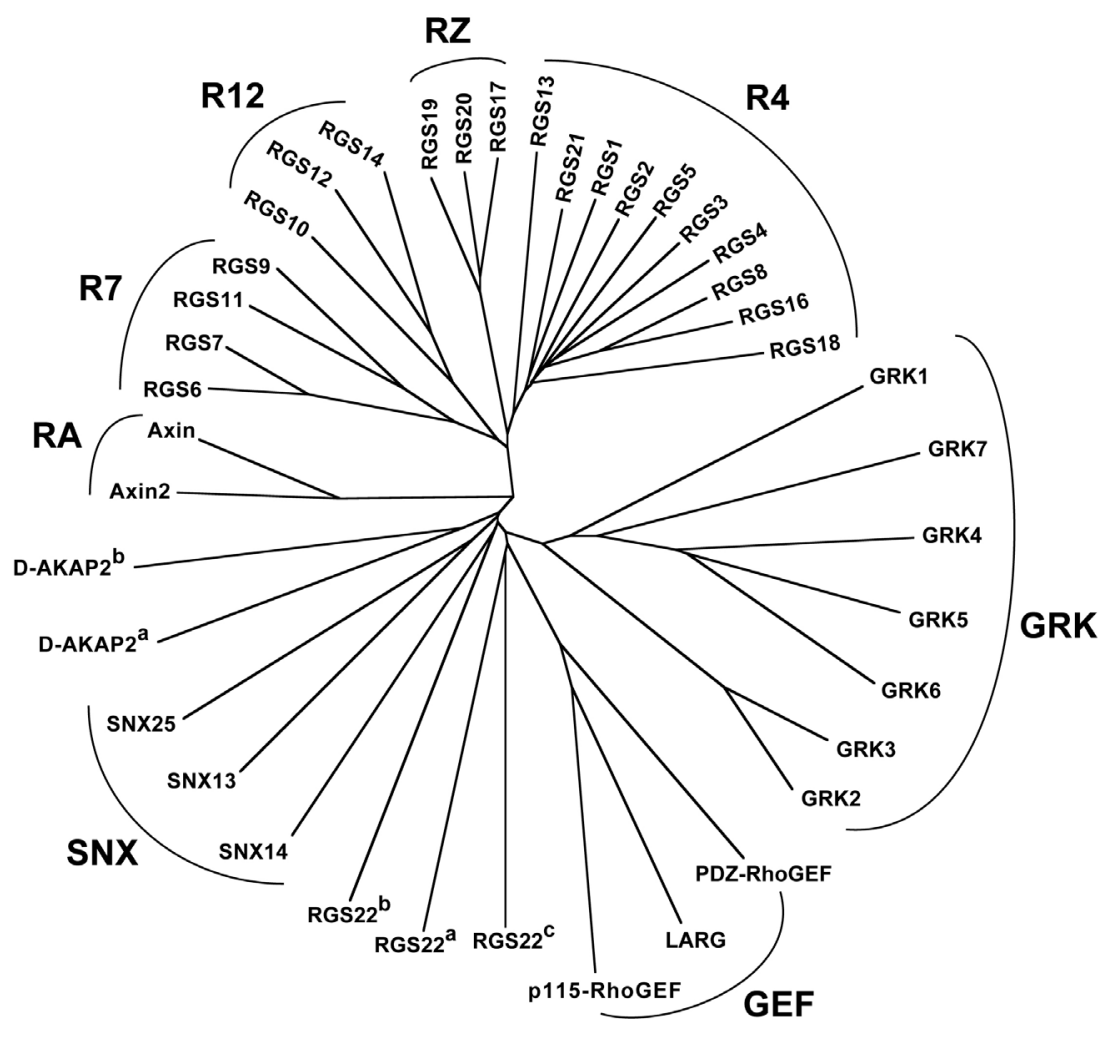
within RGS6, RGS7, and RGS11, with similarity to conventional G $\gamma$ subunits [27]. This G $\gamma$-like or "GGL" domain was subsequently shown by us

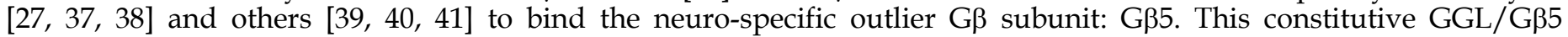
interaction was also found to hold true for the C. elegans counterparts: the R7 subfamily RGS proteins EGL-10 and EAT-

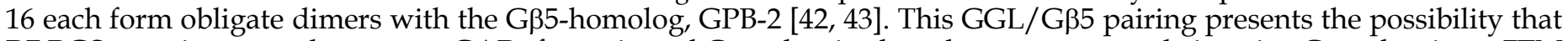

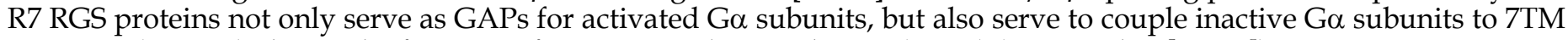
receptors (Fig. 4A) akin to the function of conventional G $\beta \gamma$ subunits (Fig. 1) (reviewed in [44, 45]). 
R7 RGS proteins also have an N-terminal DEP (Dishevelled/EGL-10/Pleckstrin homology) domain [46]. At least for the retinal-specific R7 RGS protein RGS9-1, a membrane-associated binding partner has been identified for the DEP

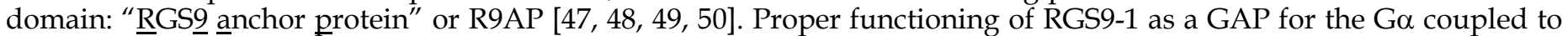
the retinal photoreceptor (rhodopsin), as well as proper membrane targetting of this GAP activity by R9AP, appear critical to normal vision; Dryja and colleagues have recently reported that loss-of-function mutations to RGS9-1 or R9AP are found in people with abnormalities in photoresponse recovery ("bradyopsia") that include an inability to see moving objects accurately, especially in low-contrast lighting, and difficulty adjusting to light intensity changes [51]. Although this is the first identified human mutation in an RGS protein causing pathological changes to the timing of 7TM signaling, we surmise that it will not be the last. Indeed, one could speculate that a component of essential hypertension might be due to loss-of-function mutation to RGS2; we and others have recently found that Rgs2-deficient mice [52] exhibit constitutive hypertension [53, 54], consistent with an earlier finding that RGS2 establishes an important negative feedback circuit on vasoconstrictive hormone signaling in vascular smooth muscle as mediated by 7TM receptors coupled to $\mathrm{Gq}$ heterotrimers [55].

\section{2.b. Membrane targeting of other RGS proteins}

With the sheer number of RGS proteins identified (e.g., at least 37 RGS proteins encoded by the human genome; Fig. 3), a central question has arisen as to how (and if) receptor selectivity is engendered for the GAP activity of specific RGS proteins. Similar to the relationship between RGS9-1 and R9AP, other mechanisms of localizing RGS proteins to membranes and/or specific 7TM receptors are being uncovered. For example, we identified an N-terminal PDZ (PSD95/Discs-large/ZO-1 homology) domain within the longest isoform of the R12 subfamily member RGS12 (Fig. 2); this PDZ domain is capable of binding peptides derived from the C-termini of 7TM receptors, including from one of the interleukin-8 receptors (CXCR2) [56, 57]. With our colleague Dr. María Diversé-Pierluissi, we have also shown that, in dorsal root ganglion neurons, the phosphotyrosine-binding (PTB) domain of RGS12 mediates its recruitment to the $\alpha 1 B$ pore-forming subunit of the N-type calcium channel $\left(\mathrm{Ca}_{\mathrm{v}} 2.2\right)$ in a neurotransmitter- and phosphorylation-dependent manner (Fig. 4B) [29]. We have since mapped the RGS12 PTB docking site to the SNARE-binding or "synprint" region of the $\mathrm{Ca}_{\mathrm{v}} 2.2$ channel (Siderovski \& Diversé-Pierluissi; manuscript submitted); the channel/PTB domain interaction, while phosphotyrosine-dependent, does not occur within a canonical Asn-Pro-X-(p)Tyr binding motif common to many PTB docking sites [58]. With the ability to interact with a multitude of proteins by virtue of its PDZ, PTB, and Ras-binding domains, along with G $\alpha$ interactions via its RGS-box and GoLoco motif (described below), RGS12 in particular appears to be a signaling nexus or 'hub' capable of coordinating signal transduction from receptor and/or non-receptor tyrosinekinases and both monomeric (Ras-superfamily) and heterotrimeric G-protein subunits [13].

An important finding in the RGS field was made by Wilkie and colleagues when they observed receptor selectivity of RGS proteins [59]. As an example, RGS1 was observed to be a 1000-fold more potent inhibitor of carbachol- than cholecystokinin-stimulated $\mathrm{Ca}^{2+}$ mobilization in pancreatic acinar cells, despite both agonists having similar coupling profiles (i.e., via $\mathrm{Gq} / 11$ family $\mathrm{G} \alpha$ subunits signaling to phospholipase C- $\beta$ ) [60]. Strikingly, the closely related RGS2, also a potent GAP for Gaq/11 family members [61], was equipotent at inhibiting carbachol and cholecystokinin signaling. The molecular determinants of receptor-selective inhibition of G-protein signaling by RGS4 have been delimited to the N-terminal 58 amino acids of this protein [59]. Full-length RGS4 and RGS4(AN58) are equipotent GAPs in vitro, however RGS4 is a 10,000-fold more potent inhibitor of muscarinic signaling in the context of pancreatic acinar cells [59].

These early findings by Wilkie et al. helped initiate the concept that domains outside the RGS-box may have significant functional effects on signaling specificity and potency. Studies using ribozyme and RNA-interference (RNAi)-mediated knockdown of endogenous RGS proteins have reinforced this notion of complementary selectivity between GPCRs and RGS proteins. For example, in studies employing A-10 rat aortic smooth muscle cells, Neubig and colleagues found that ribozyme-mediated depletion of RGS3 selectively enhances carbachol signaling via the M3 muscarinic receptor, whereas analogous depletion of RGS5 only potentiates angiotensin II signaling via the AT1a receptor; RGS2-directed ribozyme treatment had no effect on either 7TM receptor signaling pathway [62]. An obvious molecular mechanism for engendering such receptor selectivity would be direct interaction between a 7TM receptor and an RGS protein. The multidomain architecture of RGS proteins (Fig. 2) provides several potential means by which this could occur. However, to date, there is only limited evidence that 7TM receptors directly interact with RGS proteins. As previously mentioned, we found the RGS12 PDZ domain can bind peptides corresponding to the C-terminal tail of the interleukin-8 (CXCR2) 7TM receptor in vitro [56, 57], but this interaction has not yet been tested with full-length proteins in vivo. A more recent report cites a finding of in vitro binding between an intracellular loop of the M1 muscarinic receptor and RGS2 as evidence for direct coupling between the M1 receptor and RGS2 GAP activity [63]. It would be more informative if loss-of-function mutants for the M1-RGS2 interaction (i.e., mutations that do not disrupt G-protein activation or GAP activity) could be created and analyzed in a cell biological context. It must be emphasized that no report has yet demonstrated interaction between a full-length 7TM receptor and a full-length RGS protein in cells.

Our recent discovery of AtRGS1, the first plant RGS protein (from Arabidopsis thaliana), has given the clearest demonstration yet of functional linkage between an RGS-box and a particular 7TM receptor. Indeed, AtRGS1 is an amalgam of the two, with an N-terminal region predicted to have the topology and transmembrane domains of a 7TM receptor, along with a C-terminal intracytosolic RGS-box (Fig. 4C). Genetic evidence is consistent with a model that the action of the AtRGS1 RGS-box opposes that of the activated plant Ga (AtGPA1) in increasing cell elongation in hypocotyls in darkness and increasing cell production in roots grown in light [64]. In support of this model, we have shown AtRGS1 to be a potent GAP for AtGPA1 [64, 65]. The presence of both GEF-like (7TM) and GAP-like (RGS-box) domains within AtRGS1 may seem paradoxical; however, our prevailing hypothesis is that AtRGS1 represents a ligandoperated GAP, given that AtGPA1 exhibits a high rate of spontaneous nucleotide exchange, as well as slow intrinsic 
GTPase activity, in comparison to mammalian $\mathrm{G} \alpha$ subunits [65]. A definitive test of this "ligand-operated GAP" hypothesis awaits the identification of an agonist for the 7TM portion of AtRGS1. Although no ligand has yet been identified, a simple sugar appears the most likely candidate [66].

\section{2.c. RGS-box-containing RhoGEFs as G $\alpha$ effectors}

Most RGS proteins are considered negative regulators of 7TM receptor signaling, either via G $\alpha$-directed GAP activity or by "effector antagonism" (i.e., binding activated Ga GTP in competition with effectors; e.g., [67, 68, 69, 70]). In contrast, the three members of the F/GEF subfamily of RGS proteins (Figs. 2\&3), p115-RhoGEF, PDZ-RhoGEF, and leukemia-associated RhoGEF (LARG), represent positive regulators of 7TM receptor signaling - specifically, as true

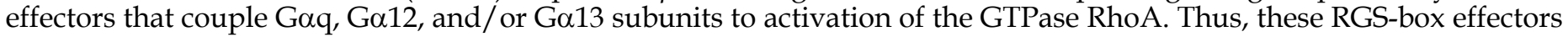
link Gq-, G12-, and G13-coupled 7TM receptors to the panoply of cytoskeletal and transcriptional responses mediated by RhoA GTP-dependent effectors [71, 72]. All three proteins possess an RGS-box N-terminal to DH (Dbl-homology) and $\mathrm{PH}$ (pleckstrin-homology) domains that, as a tandem, are responsible for catalyzing guanine nucleotide exchange necessary to convert inactive RhoA GDP into active RhoA GTP [73]. Kozasa et al. first demonstrated in 1998 that the RGS-box of p115-RhoGEF is a potent GAP for both Ga12 and Ga13 subunits [74]; more importantly, the same group found that interaction between the RGS-box and Ga13 GTP, but not Ga12 GTP, serves to trigger exchange activity by the C-terminal DH/PH cassette [75]. In contrast with p115-RhoGEF, LARG serves as a G $\alpha$-responsive RhoGEF not only

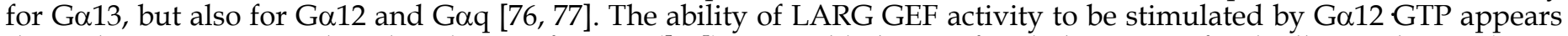
dependent on tyrosine phosphorylation of LARG ([78]), ostensibly by Tec-family kinases or focal adhesion kinase (FAK; [79]).

Recent work in PC-3 prostate cancer cells by Wang et al. suggests that these three RGS-RhoGEFs each couple distinct receptors to RhoA activation. RNAi-mediated knockdown of LARG specifically inhibited thrombin signaling via the 7TM protease activated receptor-1 (PAR1), whereas RNAi knockdown of PDZ-RhoGEF specifically inhibited lysophosphatidic acid receptor signaling; reduction of p115-RhoGEF levels had no effect on either response [80]. Of the three RhoGEFs, two of them possess an N-terminal PDZ domain: LARG and the eponymic PDZ-RhoGEF (Fig. 2). The mechanism whereby RGS-RhoGEF signaling specificity is engendered in PC-3 cells remains unknown, but might entail direct 7TM receptor tail/PDZ domain interactions. It is known that LARG associates via its PDZ domain with the Cterminal tail of the insulin-like growth factor-1 (IGF-1) receptor, providing functional linkage between extracellular IGF1 and RhoA-mediated cytoskeletal rearrangements [81, 82]. Both LARG and PDZ-RhoGEF also use their PDZ domains to bind plexin-B1 (Fig. 4D), a transmembrane receptor for the semaphorin Sema4D (a.k.a. "cluster of differentiation antigen 100" or CD100) [83]. Binding of Sema4D to plexin-B1 stimulates RhoA activation via LARG/PDZ-RhoGEF [84, $85,86,87,88$ ]. However, the role of the RGS-box in LARG/PDZ-RhoGEF signaling to RhoA activation via these latter, non-7TM receptors remains ill-defined.

Figure 4. Membrane targeting strategies employed by multi-domain RGS proteins. (A) The R7 RGS proteins form obligate

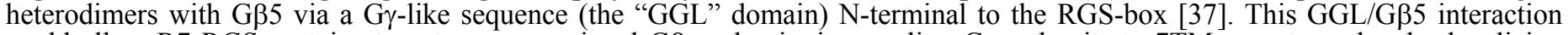
could allow R7 RGS proteins to act as conventional G $\beta \gamma$ subunits in coupling G $\alpha$ subunits to 7TM receptors, thereby localizing RGS-box-mediated GAP activity to particular receptors [44]. The DEP domain of RGS9-1 interacts with a membrane-anchoring protein (R9AP) [47]; analogous interactors may exist for the DEP domains of other R7 subfamily members [89]. (B) The PDZ domain of RGS12 is able to bind the C-terminus of the IL-8 receptor CXCR2 (at least in vitro) [57]. The RGS12 PTB domain binds the synprint ("synaptic protein interaction") region of the N-type calcium channel $\left(\mathrm{Ca}_{\mathrm{v}} 2.2\right)$; this interaction is dependent on neurotransmitter-mediated phosphorylation of the channel by Src [29]. (C) The AtRGS1 protein of Arabidopsis thaliana (thale cress) has a unique structure for an RGS protein: an N-terminus resembling a 7TM receptor and a C-terminal RGS-box [64]. Although a ligand is not known for the 7TM portion of AtRGS1, a simple sugar is most likely [66]. (D) The transmembrane receptor Plexin-B1 couples binding of the membrane-bound semaphorin Sema4D to RhoA activation via an interaction with the PDZ domain of PDZ-RhoGEF (and of the related RGS-RhoGEF LARG) [88]. Domain abbreviations [35]: IPT, immunoglobulin-like fold found in plexins, Met and Ron tyrosine kinase receptors, and intracellular transcription factors; PSI, domain found in plexins, semaphorins, and integrins; Sema, semaphorin domain.
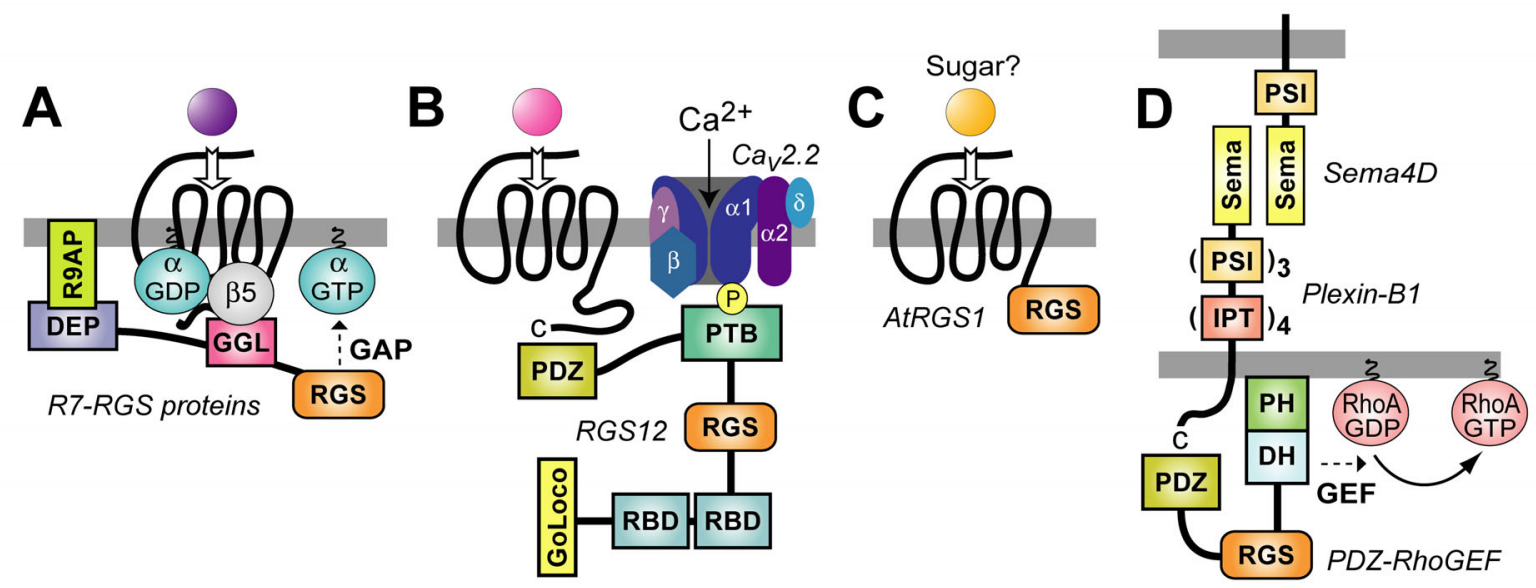


\section{Discovery of a new family of $\mathrm{G} \alpha$ regulators: The GoLoco motif proteins}

A more recent addition to the pantheon of G-protein regulatory proteins is a diverse family of G $\alpha$-interacting proteins, all of which contain one or more conserved GoLoco ("G $\alpha_{\mathrm{i} / \mathrm{o}}$-Loco" interaction) motifs (otherwise known as GPR or "G-protein regulatory" motifs) (Fig. 5A) [28, 90]. GoLoco motif-containing proteins generally bind to GDPbound $\mathrm{G} \alpha$ subunits of the $\mathrm{G}_{\mathrm{i}}$ (adenylyl-cyclase inhibitory) class and act as guanine nucleotide dissociation inhibitors (GDIs), slowing spontaneous exchange of GDP for GTP and inhibiting association with G $\beta \gamma$ subunits [91]. Our determination of the crystallographic structure of $\mathrm{Ga}_{\mathrm{i} i}$ GDP in complex with the 36 amino-acid GoLoco motif of RGS14 [92] revealed critical determinants of G $\alpha$ subunit specificity and GDI activity. The N-terminal alpha-helix of the GoLoco peptide was seen sandwiched between switch II and the $\alpha 3$ helix of the $\mathrm{G}_{\mathrm{i} 1}$ Ras-like lobe (Fig. 5B), grossly deforming the normal site of $G \beta \gamma$ interaction [92]. The highly conserved aspartate-glutamine-arginine triad, which defines the final residues of the highly-conserved 19 amino-acid GoLoco signature (Fig. 5A), was found to orient the arginine residue into the guanine nucleotide binding pocket of $\mathrm{G} \alpha$, allowing contacts to be made between its basic $\delta$-guanido group and the $\alpha$ - and $\beta$-phosphates of GDP (Fig. 5B). Loss of GDI activity results from mutation of this arginine [92, 93], or of the preceding aspartate which aids its orientation [94].

Figure 5. The GoLoco motif, a G⿰ $\alpha_{\mathrm{i} / 0}$-interacting polypeptide found singly or in arrays in various proteins, binds across the face of Ga. (A) Alignment of the highly-conserved, 19 amino-acid GoLoco motif signatures from human RGS12 and RGS14 is colored according to side-chain chemistry using Clustal-X defaults [33]. Consensus symbols for amino-acid character are hyphen (-), acidic; $\Phi$, hydrophobic; $\Psi$, large aliphatic; $(+)$, basic; and $\pi$, small side chain. Domain abbreviations: RapGAP, Rap-specific GTPase-activating protein domain. (B) Our structure of $\mathrm{G \alpha}_{\mathrm{i} 1}$ (green with translucent space-filling shell; switch regions in blue) bound to a 36-amino acid peptide derived from the GoLoco motif-region of RGS14 [92]. The GoLoco peptide (yellow) binds across both Ras-like and all $\alpha$-helical lobes within $\mathrm{G \alpha}_{\mathrm{i}}$, trapping GDP (brown) within its binding site. Note the relative position of the GoLoco triad's arginine "finger" (yellow in ball-and-stick representation) reaching into the GDP binding site. G⿰ $\alpha_{i 1}$-specific contacts from the $\alpha$-helical lobe to the C-terminal portion of the GoLoco peptide are denoted in magenta.

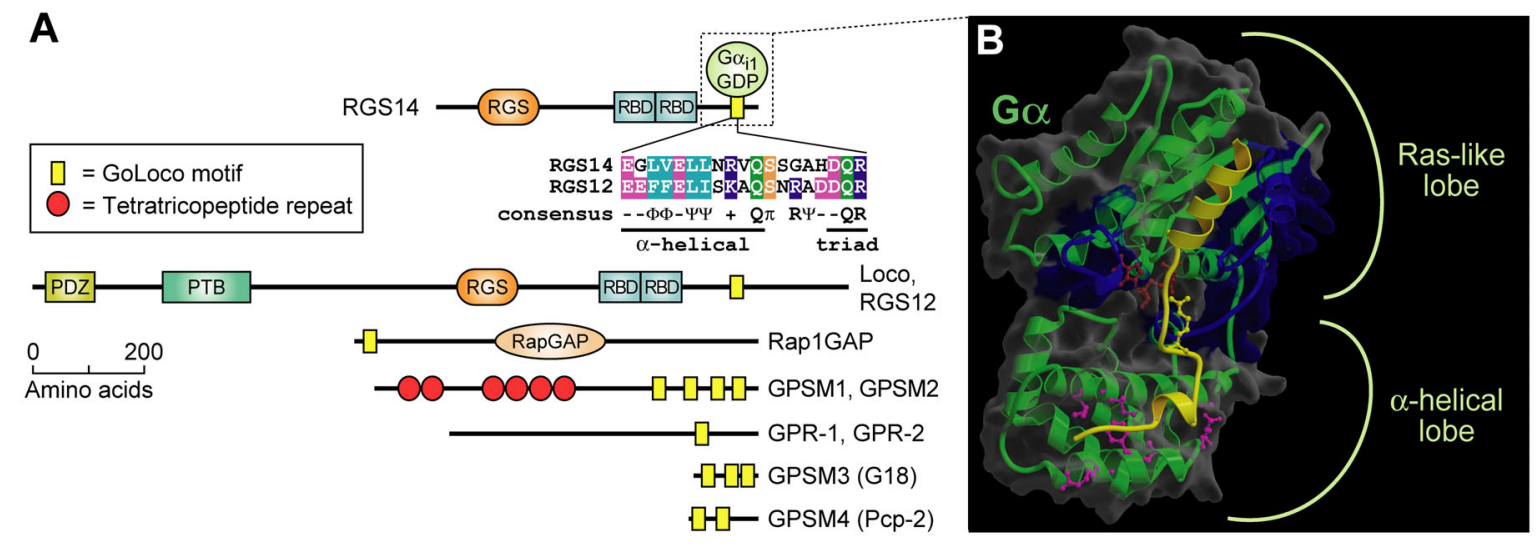

The family of known GoLoco motif-containing proteins represents a diverse set of signaling regulators, each first discovered and characterized in vastly different contexts; accordingly, their nomenclature is often misleading or uninformative. Here we describe these proteins using their newly-approved Human Genome Organisation (HUGO) nomenclature (http:// www.gene.ucl.ac.uk/nomenclature/). One subset of GoLoco proteins includes the R12 subfamily of RGS proteins: RGS12, RGS14, and the Drosophila ortholog Loco. We originally cloned both RGS12 and RGS14 in 1997 from rat C6 glioblastoma cells using degenerate PCR primers directed to sequences conserved within all RGS-box domains [57, 95]. In addition to a hallmark RGS-box and tandemly-repeated Ras-binding domains, each of these R12subfamily members contains a single, C-terminal GoLoco motif that is a GDI for Go $\alpha_{i}$ subunits [96, 97]. Concomitant with our discovery of RGS12 and RGS14, the latter protein was also isolated as a putative effector for Rap2 GTP in a yeast two hybrid screen for Rap2-interacting proteins [98]. A second subset of GoLoco proteins, consisting of the G-protein signaling modulator protein GPSM1 (formerly AGS3 [90]), GPSM2 (formerly LGN [99]), and the Drosophila ortholog Partner of Inscuteable (Pins/Rapsynoid; [100, 101]), all contain a tandem array of three to four GoLoco motifs at their Cterminus, along with an N-terminal region of multiple tetratricopeptide repeats (TPRs; Fig. 5A). The Caenorhabditis elegans proteins GPR-1 and GPR-2 are distantly related (Fig. 6) to GPSM1, GPSM2, and Pins, and have only one easilyidentifiable GoLoco motif [102, 103, 104]. A third subfamily consists of GPSM3 (formerly G18 [94]), and Purkinje cell protein-2 (Pcp-2, a.k.a. GPSM4 [105]); both are small proteins that contain between one and three GoLoco motifs (depending on alternative splicing), but no other identifiable functional domains. The sole member of the fourth and final subset of GoLoco proteins is Rap1GAP. With a few limited exceptions, all the aforementioned GoLoco-containing proteins demonstrate GDP-dependent interaction with $\mathrm{G}_{\mathrm{i} / \mathrm{o}}$ subunits and function as GDIs for G $\alpha$ subunits. 
Figure 6. Relationship between GoLoco motif sequences from all GoLoco proteins identified to date. Unrooted dendrogram was generated by Clustal-W [33] and TreeView [34] using GoLoco motif sequences originally published in our comprehensive review of the GoLoco motif [91]. The individual GoLoco motifs from tandem arrays (Fig. 5A) are numbered GL1 to GL4 starting from the first N-terminal motif. Species abbreviations: a, Anopheles gambiae (mosquito); Cb, Caenorhabditis briggsae; Ce, Caenorhabditis elegans; d, Drosophila; f, Fugu rubripes; h, human; m, mouse; r, rat; z, Danio rerio (zebrafish). Note that there are two LGN-like proteins encoded by the genome of Fugu rubripes; we have denoted the one more closely-related to mammalian LGN/GPSM2 as fGPSM2 (Ensembl translation ID SINFRUP00000160450) and the more distantly-related protein as fLGN2 (Ensembl SINFRUP00000146023). Two conclusions can immediately be drawn from this analysis: (i) Drosophila Pins, which has only three GoLoco motifs (Fig. 8) vs the four motifs of GPSM1 and GPSM2 proteins, has lost the second motif, since dPins GL2 is most similar to GL3 of GPSM1/2 proteins, and dPins GL3 is most similar to GL4 of GPSM1/2 proteins; (ii) the triple GoLoco motif protein GPSM3 (a.k.a. G18) is not directly related to the tandem GoLoco arrays of GPSM1/2 proteins (i.e., GPSM3 is not merely missing the N-terminal TPR region of GPSM1/2).

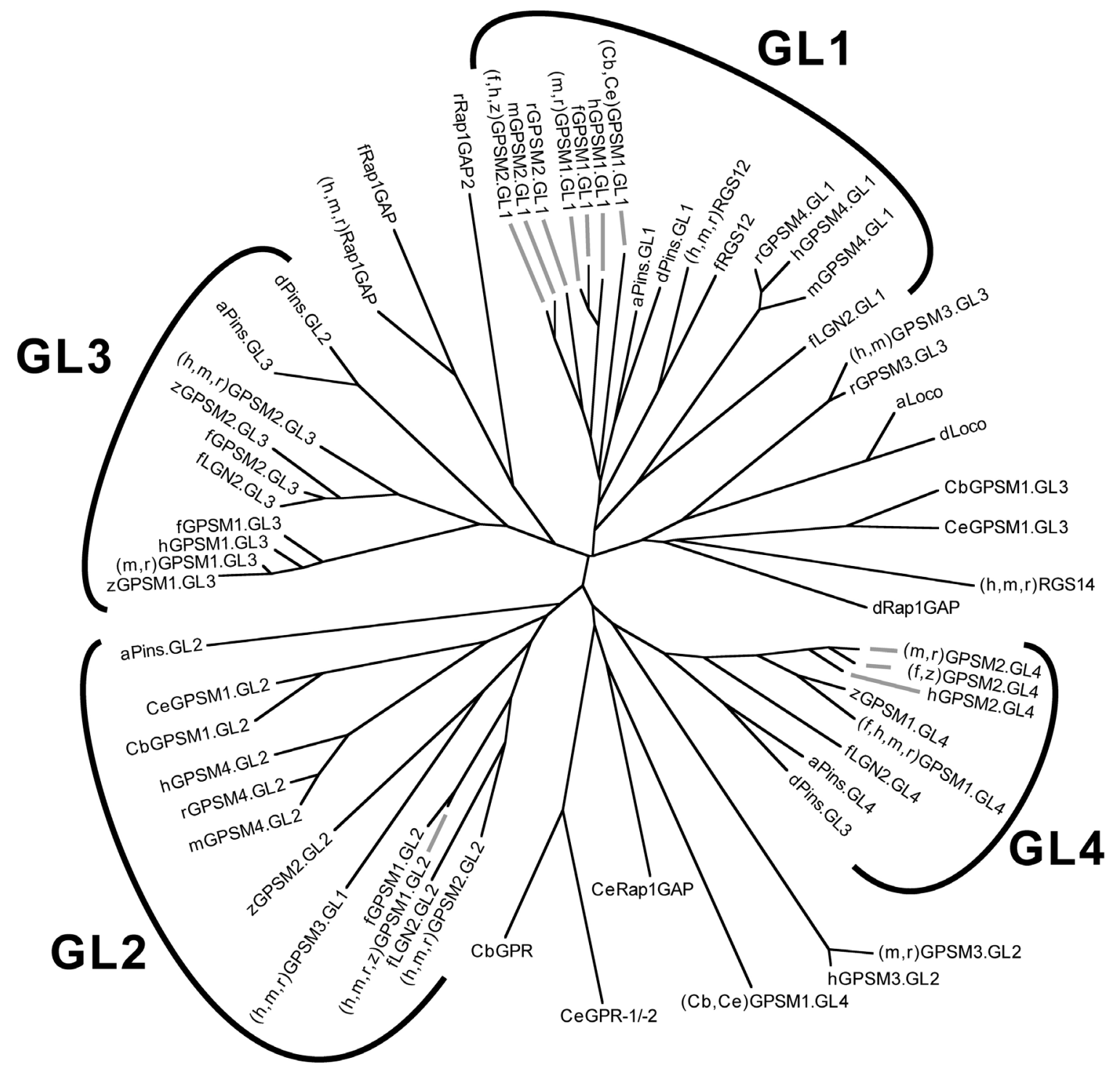

Currently, the only compelling evidence that a GoLoco motif-containing protein acts within the framework of canonical GPCR signaling (outside the R12-subfamily RGS proteins; Fig. 4) has been provided by studies of Rap1GAP as a potential effector for $\mathrm{G} \alpha_{\mathrm{i} / \mathrm{o} / \mathrm{z}}$ subunits. The Rap1GAPII isoform, originally described by Mochizuki and colleagues [106], contains a 31 amino-acid N-terminal extension encoding a full GoLoco motif. In vivo characterization of Rap1GAPII suggested that the GoLoco-containing region specifically interacts with constitutively-activated, GTPasedeficient $\mathrm{G} \alpha_{\mathrm{i}}(\mathrm{Q} 205 \mathrm{~L})$ [106]. In contrast, in vitro studies indicated a strong preference for interaction with GDP-bound $\mathrm{G} \alpha_{\mathrm{i}}$

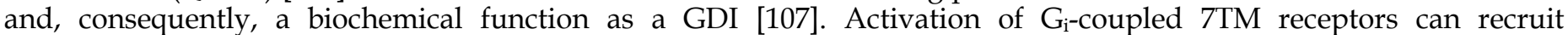
Rap1GAPII to the plasma membrane to accelerate GTP hydrolysis by Rap1. Deactivation of Rap relieves an inhibitory constraint on Ras, thus promoting activation of the MAPK pathway [106]. Similarly, G $\alpha_{z}$-coupled 7TM receptors also employ Rap1GAPI to attenuate Rap1-mediated ERK activation and PC12 cell differentiation [108, 109]. However, some discrepancies exist between these reports describing GTP-dependent G $\alpha$ /Rap1GAP interactions and a report showing that $\mathrm{G \alpha}_{0}$-GDP interacts with Rap1GAP to block Rap-directed GAP activity [110]. It may be that Ga/Rap1GAP interactions are contextually determined by cell-type and splice-form, and also that multiple Ga-binding sites might exist on Rap1GAP isoforms. Nonetheless, these studies indicate that, at least in the cell transfection paradigm, the 
GoLoco protein Rap1GAP can modulate 7TM receptor signaling; however, the field requires future studies to further delineate the mechanisms of GoLoco-mediated regulation of 7TM receptor signal transduction.

In sharp contrast to the studies on Rap1GAP, there is a paucity of evidence supporting GoLoco motif-mediated modulation of 7TM receptor signaling in physiologically-relevant systems. Results from our lab and from others indicate that GPSM1-4 proteins do not appear to modulate conventional aspects of 7TM receptor signal transduction. That is, gross overexpression of GPSM1-4 proteins does not alter either the kinetics or magnitude of receptor-induced effector activation. We interpret this to indicate that many GoLoco motif proteins may be spatially segregated from interacting with plasma membrane-delimited 7TM receptor signaling components. Indeed, Graber and colleagues demonstrated this using Sf9 insect-cell membranes overexpressing GPSM1 and the 5- $\mathrm{HT}_{1 \mathrm{~A}}$ serotonin receptor [111]. Membrane-bound GPSM1 did not affect receptor coupling, but adding soluble GPSM1 protein to the membrane preparation attenuated high affinity agonist binding. A recent report indicates that overexpression of GPSM1 in COS-7 cells may have a modest effect on adenylyl cyclase sensitization and $\mathrm{G} \alpha_{\mathrm{i} 3}$ subunit stability [112]; however, the physiological relevance of this phenomenon remains to be elucidated. In this light, a recent report indicates that GPSM1 is upregulated in the rat prefrontal cortex following withdrawal from repeated cocaine administration [113]. Evidence suggests that GPSM1 modulates cocaine-induced plasticity in 7TM receptor signaling, as increased GPSM1 expression correlates with attenuated $\mathrm{G}_{\mathrm{i}}$-coupled receptor signal transduction [113]. Thus, rather than having a role as acute elements of G-protein signal modulation, the GPSM proteins may function as G $\alpha$ 'sinks' to regulate the overall availability and stability of the Ga component of the heterotrimeric G-protein cycle.

Figure 7. Experimental paradigm used to demonstrate that GoLoco motif-derived peptides can uncouple heterotrimeric Gprotein signaling, but have no intrinsic ability to directly activate $\mathbf{G} \beta \gamma$-dependent signaling. (A) The dopamine agonist quinpirole, when applied to AtT-20 cells stably or transiently expressing the D2-dopamine receptor [116], activates Gi-heterotrimers

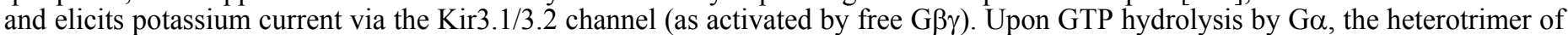

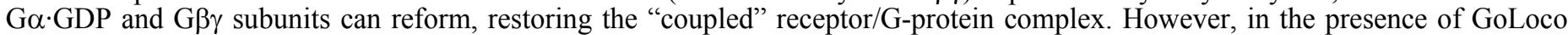

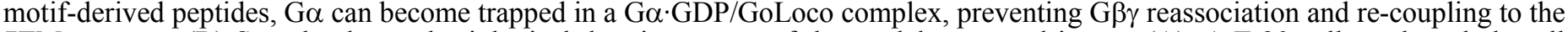
7TM receptor. (B) Sample electrophysiological data in support of the model presented in part (A). AtT-20 cells under whole-cell patch-clamp configuration to measure potassium currents were treated with repeated quinpirole pulses (orange circles) or buffer (open circles) after loading with $10 \mu \mathrm{M}$ RGS12 GoLoco peptide (left panel) or control scrambled peptide (right panel).
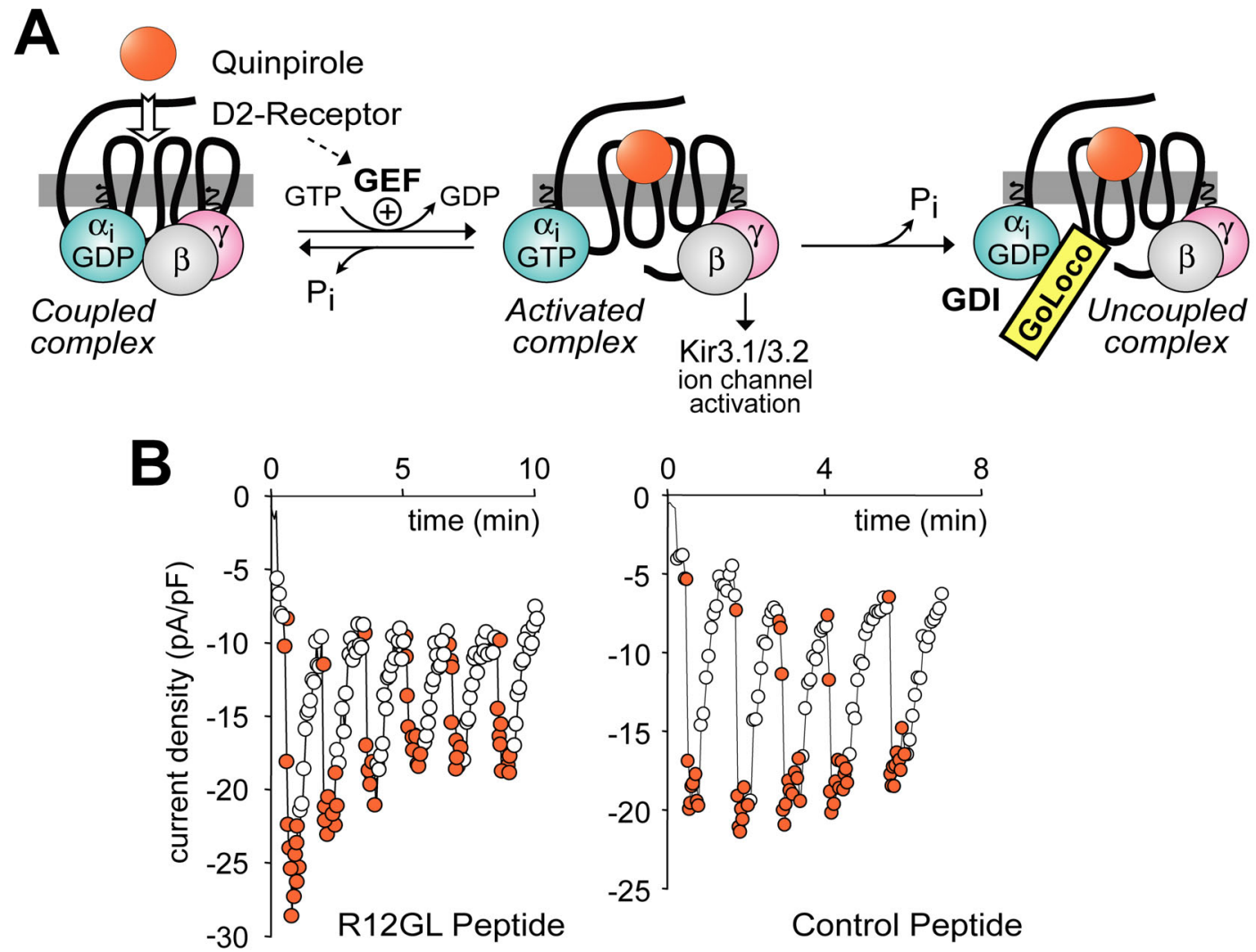

With a view to placing GoLoco motif-containing proteins within the classical paradigm of 7TM receptor signal transduction, it has been suggested that GoLoco motifs may activate heterotrimeric G-protein signaling by actively displacing $G \beta \gamma$ from $G \alpha[90,100,114]$. Indeed, Smrcka and colleagues have demonstrated that a GoLoco consensus peptide can actively displace $G \beta \gamma$ from a preformed heterotrimer, although at a rather high 20,000-fold molar excess in vitro (i.e., $1 \mu \mathrm{M}$ GPSM1 consensus peptide mixed with $50 \mathrm{pM} \mathrm{G \alpha \beta \gamma}$ ) [115]. However, using similar levels of GoLoco peptides $(10 \mu \mathrm{M})$ microinjected into cells, it appears that GoLoco proteins only have access to G $\alpha$ GDP following a cycle of receptor-mediated GEF activity. In a recent study, Oxford and colleagues measured $\mathrm{G}_{\mathrm{i}}$-coupled activation of $\mathrm{Kir}_{3.1 / 3.2}$ potassium channels (a $G \beta \gamma$-mediated process; Fig. 7A) to interrogate the ability of GoLoco peptides to promote $G \beta \gamma$ signaling [116]. Electrophysiological recordings of potassium channel currents activated via D2-dopamine receptor 
stimulation were made in AtT-20 cells. Intracellular application of GoLoco peptides derived from RGS12 and GPSM1 had little effect on single agonist application-induced channel activation (e.g., sample data in Fig. 7B; C.K. Webb, C.R. McCudden, F.S. Willard, R.J. Kimple, D.P. Siderovski, G.S. Oxford; manuscript submitted). However, repeated applications of the D2-receptor agonist quinpirole produced progressive uncoupling of receptor and effector (e.g., Fig. 7B). Repeated activation of potassium current in the same cells using somatostatin, a $\mathrm{G}_{0}$-coupled 7TM receptormediated response, was unaffected by GoLoco peptide application, consistent with the G $\alpha_{\mathrm{i}}$-selective nature of the GoLoco peptides employed. These results indicate that, if GoLoco motifs do have a functional role in modulating 7TM receptor signaling, it may be more subtle to discern than has previously been appreciated.

\section{GoLoco motif proteins and a novel $\mathbf{G} \alpha$ nucleotide cycle involved in cell division}

Although the GoLoco motif was first discovered in the context of 7TM receptor signaling, a central role is emerging for GoLoco motif-containing proteins in an unexpected arena: the control of mitotic spindle organization, microtubule (MT) dynamics, and the act of chromosomal segregation during cell division. Early evidence supporting a connection between $\mathrm{G} \alpha$ subunits, GoLoco proteins, and MT dynamics has arisen from studies of asymmetric cell division (ACD) in model organisms.

\section{4.a. G $\alpha$ and GoLoco protein involvement in Drosophila asymmetric cell division}

One of the first observations that placed a GoLoco protein within the machinery of cell division was the finding that a complex between G $\alpha$ and the multi-GoLoco protein Partner of Inscuteable (Fig. 8) is crucial for dictating ACD in Drosophila neuroblasts [100, 101]. Upon delamination from ventral neuroectoderm, these neuroblast cells adopt an apical-basal axis of polarity and divide asymmetrically along this axis to produce a large apical neuroblast competent for further ACD and a small ganglion mother cell committed to differentiation (reviewed in [117]). In this process, apical determinants Bazooka (PAR3), PAR6, and atypical protein kinase C (aPKC) form a protein complex that recruits the Drosophila protein Inscuteable (dInsc) to the apical cortex [118, 119]. Loss of inscuteable expression leads to mislocalization of basal determinants Numb and Prospero, as well as misorientation of the mitotic spindle and, thus, randomization of the plane of cell division with respect to the apical-basal axis [120].

Deletional analysis of dInsc has defined a central Armadillo repeat-containing "asymmetry domain" sufficient to mediate all known functions of the full-length protein [121, 122]. In searching for asymmetry domain-interacting proteins as potential dInsc effectors, three groups independently discovered the GoLoco motif-containing Partner of Inscuteable (Pins) protein [100, 101, 123]. Pins is the archetypal member of a conserved class of TPR and GoLoco-motif containing proteins involved in cell division processes (GPSM1, GPSM2, Pins; Fig. 5A). dInsc, Pins, and Drosophila Gai form an apical protein complex essential for neuroblast ACD; ablation of maternal and zygotic Pins results in defective spindle orientation, a failure to segregate determinants asymmetrically, and a limited asymmetry in neuroblast division $[100,101,123]$. Similarly, overexpression of wildtype Gai inhibits polarization of asymmetry determinants and causes mitotic spindle misorientation [124]. However, overexpression of constitutively-active (i.e., GTPase-deficient) Gai(Q205L) has essentially no effect on neuroblast ACD. Thus, in the specific example of Drosophila neuroblast ACD, the functional $\mathrm{G} \alpha$ species appears not to be GTP-bound Gai (the active form in 7TM receptor signaling; Fig. 1), but rather

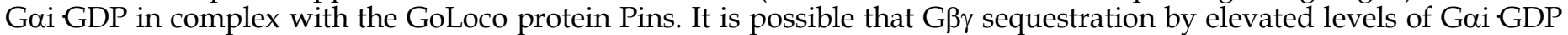
leads to the observed aberrations in neuroblast ACD. However, significant differences are seen in daughter cell size and cell-fate determinant mislocalization upon Gai overexpression versus loss of G $\beta 13 F$ (ortholog of the mammalian G $\beta$ subunits Gß1-4) [124, 125]. It is also important to note that the Insc/Pins/Gai GDP complex isolated from Drosophila neuroblasts is devoid of G $\beta 13 F$ [124], consistent with the mutually-exclusive nature of GoLoco- vs G $\beta \gamma$-binding to Ga GDP.

Figure 8. Known interactions between apical polarity proteins PAR3, PAR6, atypical PKC (aPKC), and Drosophila Inscuteable (dInsc), Partner of Inscuteable (Pins), and Gai in Drosophila neuroblast asymmetric cell division. ARM, homology to Armadillo repeats. Red circles, TPR motifs; yellow rectangles, GoLoco motifs.

\section{4.b. Involvement of G $\alpha$ and GoLoco proteins GPR-1/-2 in $C$. elegans asymmetric cell division}

The involvement in ACD of many of these polarity proteins is conserved throughout metazoan organisms [91, 126]. Early evidence that $\mathrm{G} \alpha$ subunits also function in ACD in the nematode worm C. elegans came from studies in which simultaneous RNA

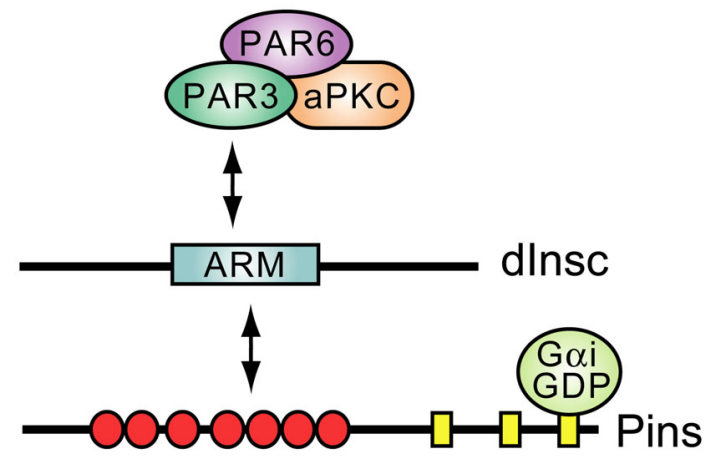
interference (RNAi)-mediated knockdown of two C. elegans Ga subunits, GOA-1 (a G $\alpha_{0}$ homolog) and GPA-16 (a Ga $\alpha_{i}$ homolog), was found to cause a mitotic spindle positioning defect resulting in symmetric division of the nematode zygote [127]. Normally, division of the one-cell stage $\left(\mathrm{P}_{0}\right)$ C. elegans embryo is asymmetric, yielding a large anterior $(A B)$ blastomere and a smaller posterior blastomere $\left(\mathrm{P}_{1}\right)$. The mitotic spindle at metaphase of this first division is symmetrically positioned along the anterior-posterier axis, but during anaphase the anterior centrosome remains fixed while the posterior centrosome moves toward the posterior cortex with a characteristic rocking motion. These events are believed to originate from asymmetric forces acting through astral MTs that radiate from the mitotic spindle poles to the cell cortex.

Grill et al. recently employed UV laser-induced centrosome disintegration on one-cell C. elegans embryos to study the basis of this net force imbalance [128]; calculations based on observed speeds of centrosome fragmentation led Grill 
et al. to conclude that the force imbalance results from a larger number ( $\sim 50 \%$ more) of force generators in the posterior cortex pulling on astral MTs (relative to the anterior cortex), rather than an increase in the magnitude of force derived from each force generator. In these studies, RNAi-mediated knockdown of GOA-1 and GPA-16 was found to greatly reduce aster fragmentation speeds upon laser-induced centrosome disintegration [128], suggesting that some form of $\mathrm{G} \alpha$ signaling during C. elegans ACD is required to mediate posterior astral MT force generation.

A functional genomic screen for genes regulating early C. elegans embryo cell division by Gönczy and colleagues identified the Pins-related GoLoco proteins GPR-1 and GPR-2 (Fig. 5A \& 9) [129]. GPR-1 and -2 are essential for establishing proper pulling forces during asymmetric $P_{0}$ division in C. elegans [102]. RNAi-mediated reduction in expression levels of these two, near-identical GoLoco proteins leads to a spindle positioning defect indistinguishable from that seen in goa-1/gpa-16 (RNAi) embryos [102, 127]. In laser-mediated spindle-severing studies, Colombo et al. established that, during normal asymmetric division, the peak velocity of the posterior spindle pole is $\sim 40 \%$ greater than that of the anterior one, presumably reflecting a larger net pulling force by astral MTs [102]. In contrast, anterior and posterior spindle pole velocities are both equal and greatly reduced in gpr-1/-2 (RNAi) embryos and in goa-1/gpa-16 (RNAi) embryos [102]. Immunofluorescence studies revealed $\sim 50 \%$ more GPR-1/-2 protein on the posterior cortex; this asymmetric cortical distribution of GPR-1/-2 is dependent on PAR-3 function [102], and nicely correlates with Grill's calculation of $\sim 50 \%$ more force generators at the posterior cortex [128] (described above), suggesting that GPR-1/-2 may specify motor localization and/or directly transact a function required for force generation on astral microtubules during chromosomal segregation. These findings by Colombo et al. that the GoLoco proteins GPR-1 and GPR-2 are critical to C. elegans ACD are wholly consistent with independent reports published simultaneously [103, 104].

Figure 9. Alignment of $\boldsymbol{C}$. elegans GPR-1 and GPR-2 protein sequences. Although individual tetratricopeptide repeats (TPRs) are not easily identified within the N-termini of GPR-1 and GPR-2 (as they are within mammalian and Drosophila homologs; Figs. 5A \& 8), amino-acids 14 to 341 (boxed in grey) are predicted by protein-fold recognition algorithms (e.g., ref. [36]) to fold into a right-handed alpha-alpha superhelix (SCOP identifier d1ld8a ; expect value $5 \times 10^{-5}$ ) similar to folds exhibited by TPR-containing proteins [130]. Also boxed is the conserved GoLoco motif signature (amino-acids 425-442) and its consensus as defined in [91]. Consensus symbols for amino-acid character are hyphen (-), acidic; $\Phi$, hydrophobic; $\Psi$, large aliphatic; $(+)$, basic; and $\pi$, small side chain.

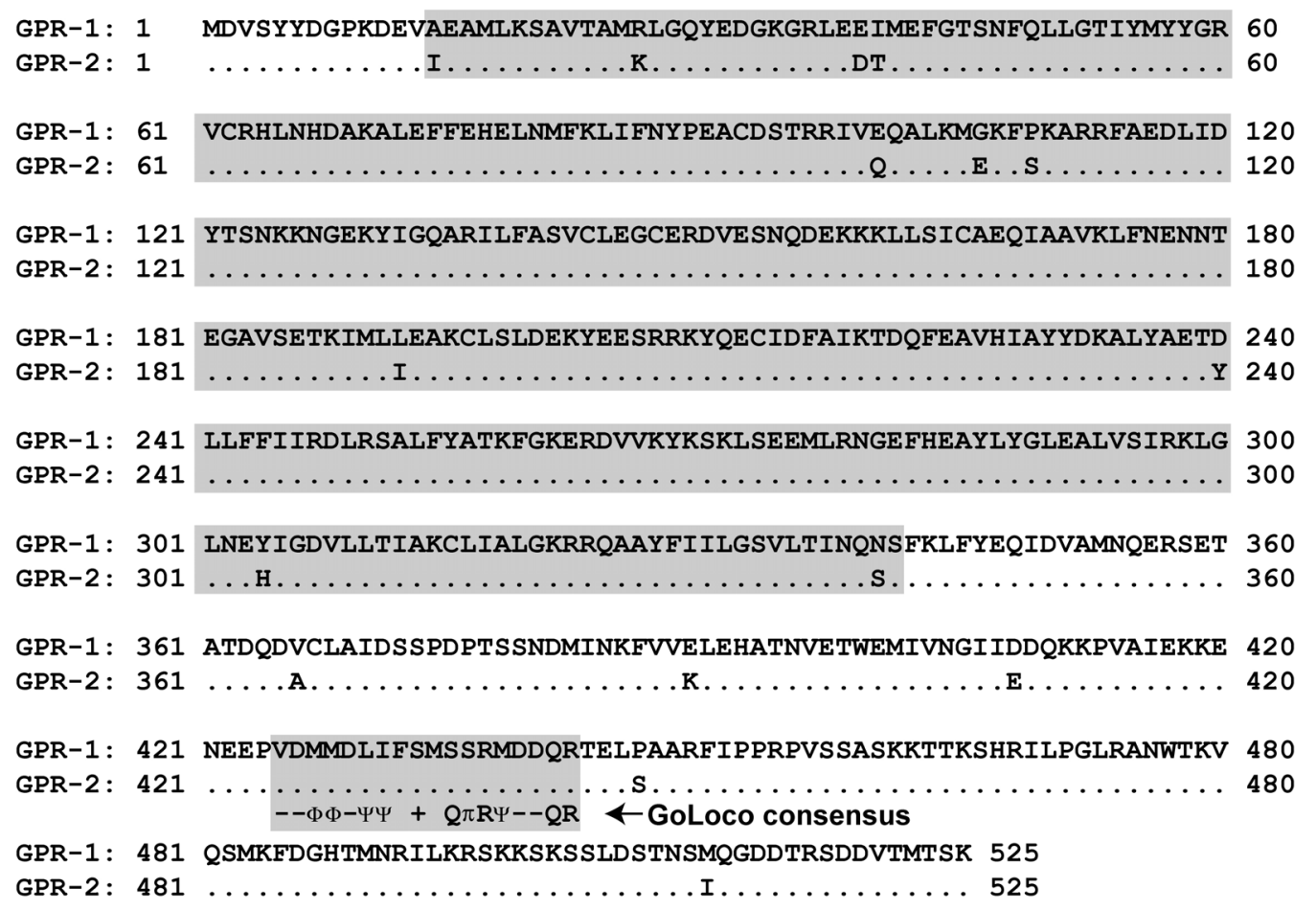

\section{4.c. G $\alpha$-directed GEF and GAP activities implicated in $C$. elegans asymmetric cell division}

Not only are G $\alpha$ subunits (GOA-1, GPA-16) and GoLoco proteins (GPR-1, GPR-2) required for asymmetric $P_{0}$ division in C. elegans, but other novel regulators of the $G \alpha$ guanine nucleotide cycle have also emerged from genetic studies as being critical to this process. For example, Miller [131, 132] first isolated a novel gene ric-8 ("resistance to inhibitors of cholinesterase", a.k.a. Synembryn) as an upstream regulator of the C. elegans G $\alpha_{\mathrm{q}}$-like protein EGL-30. ric-8 reduction-of-function mutants $(m d 303, m d 1909)$ were found to have high rates of embryonic lethality, which could be augmented to almost $100 \%$ in embryos heterozyous for goa-1 loss-of-function alleles, thus suggesting that RIC-8 and GOA-1 proteins signal in the same pathway during embryogenesis [133]. Reduced posterior centrosome rocking during the $\mathrm{P}_{0}$ cleavage was observed in ric- 8 mutant embryos, as well as loss of mitotic spindle and daughter blastomere asymmetries. Most compelling is that these ric- 8 mutant phenotypes are reminiscent of those seen in goa-1/gpa-16 (RNAi) embryos. 
It is believed that RIC-8 may represent a novel, receptor-independent guanine nucleotide exchange factor (GEF) for $\mathrm{G} \alpha$ subunits. Tall et al. recently isolated the rat paralogues Ric-8A and Ric-8B in a yeast two-hybrid screen for $\mathrm{Ga}_{\mathrm{s}}$ - and $\mathrm{G} \alpha_{\mathrm{o}}$-interacting proteins [134]; their initial biochemical analyses indicated that rat Ric-8A can act as a GEF in vitro for several mammalian $\mathrm{G} \alpha$ subunits, including $G \alpha_{i 1}, G \alpha_{o}$ and $G \alpha_{q}$. The GEF activity of rat Ric-8A was found to have characteristics distinct from those of 7TM receptors; specifically, Ric-8A appears to interact preferentially with isolated G $\alpha$ GDP subunits to accelerate the release of GDP by G $\alpha$, the rate limiting step in the G-protein cycle [135]. It is important to stress that, in this initial study, Ric-8A was found to be unable to catalyze the loading of GTP onto

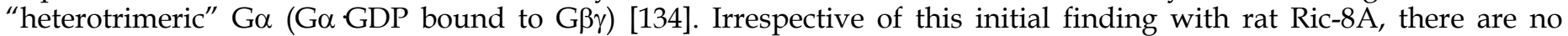
published reports that the C. elegans RIC-8 protein can act as a GEF for the nematode G $\alpha$ subunits GOA-1 or GPA-16. More importantly, it has not been determined whether mammalian Ric-8A or C. elegans RIC-8 can act as a GEF for GoLoco-bound G $\alpha$ GDP subunits. The Ric-8 gene is highly evolutionarily conserved, but the encoded protein has no obvious domain or motif structure. We have observed Ric-8 orthologues in the Drosophila, mouse, and human genomes (GenBank NP_572550, NP_444424, and NP_068751, respectively). It is tempting to speculate that deletion of (or loss-offunction mutations in) Drosophila Ric-8 will lead to severe phenotypes affecting neuroblast ACD. Similarly, it will be important to examine the physiological roles of Ric-8A and Ric-8B in mammals. The field of mammalian asymmetric cell division is underdeveloped in comparison to C. elegans and Drosophila. Nonetheless there are increasingly powerful approaches being used to study this process in the developing mouse zygote [136] and nervous system [137] that should be employed to investigate the roles of these novel G $\alpha$ regulators in ACD.

Timely deactivation of G $\alpha$ GTP may also be required for proper $P_{0}$ division in C. elegans. Koelle and Hess have discovered that $\mathrm{rgs}-7$, one of the 13 C. elegans RGS genes (rgs-1 to -11, eat-16, egl-10), is intimately involved in asymmetric $\mathrm{P}_{0}$ division. Loss of $r g s-7$, encoding a potential GAP for both GOA-1 GTP and GPA-16 GTP, results in overly vigorous posterior centrosome rocking (H. Hess \& M. Koelle, personal comm.; reviewed in [91]). Their finding implies that elevated G $\alpha$ GTP levels, presumably by loss of RGS-7 GAP activity, may result in excess force by astral MTs and further implies that, at least in the nematode, Ga GTP may be the active species for force generation. These genetic and RNAibased studies have identified $\mathrm{G} \alpha$ subunits and three novel $\mathrm{G} \alpha$ regulatory activities (GDI, GEF, and GAP proteins) as having critical roles in the process of $C$. elegans ACD, as summarized in Fig. 10A. Thus, initial findings from both Drosophila and C. elegans genetics suggest that $\mathrm{G} \alpha$ subunits and novel regulators of their guanine nucleotide cycle play critical and evolutionarily-conserved roles in establishing appropriate astral MT force on the mitotic spindle for proper chromosomal segregation. Collectively, this initial evidence suggests a preliminary working model in which productive cycling of $\mathrm{G \alpha}_{\mathrm{i}}$ subunits between GDP- and GTP-bound states, established through the actions of GoLoco GDI, Ric-8 GEF, and RGS-box GAP activities, is required for mitotic spindle organization and function.

Figure 10. A. Summary of data implicating $G \alpha$ and its regulators in generation of asymmetric anterior/posterior forces acting on the mitotic spindle of one-cell $C$. elegans zygotes during ACD (reviewed in [91]). B. Model of a novel Ga nucleotide cycle incorporating the data of part A. RGS-7 contains N-terminal homology to conserved region 2 ("C2") of protein kinase C and an RGS-box presumed in the model to exert GAP activity. Force arrow denotes speculation that, at least in the nematode, the GTPbound form of GOA-1/GPA-16 interacts, directly or indirectly, with astral MTs to generate force on the mitotic spindle.

\begin{tabular}{|c|c|c|}
\hline & Deletion/RNAi & Phenotype \\
\hline $\mathbf{G} \alpha$ & GOA-1/GPA-16 & $\downarrow$ Force \\
\hline $\mathbf{G} \propto$ GDI & GPR-1/GPR-2 & $\downarrow$ Force \\
\hline $\mathbf{G} \alpha \mathbf{G E F}$ & $\mathrm{RIC}-8$ & $\downarrow$ Force \\
\hline $\mathbf{G} \propto \mathbf{G A P}$ & RGS-7 & $\uparrow$ Force \\
\hline
\end{tabular}

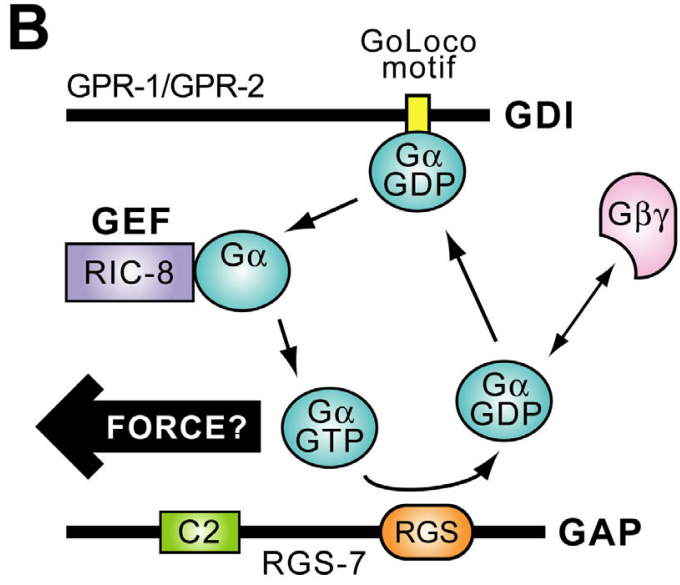

\section{4.d. A novel G $\alpha$ nucleotide cycle?}

In establishing an initial model of $\mathrm{G} \alpha$ nucleotide cycling underlying ACD that explains the genetic evidence (Fig. 10B; [91, 103]), several assumptions and accommodations have been made that demand verification by biochemical studies. For example, the biochemical actions on isolated $\mathrm{G} \alpha$ subunits currently assumed for GoLoco proteins and for RIC-8 are actually diametrically opposed: GDI activity preventing nucleotide release vs GEF activity promoting nucleotide release, respectively. Yet these opposing biochemical activities, when individually removed by RNAi or lossof-function mutation, result in the same nematode zygote phenotype of decreased force and symmetric division (Fig. 10A). To accommodate this apparent enigma, we and others propose that RIC-8 requires interdiction by the GPR-1/-2 GoLoco motif to function on GOA-1 and/or GPA-16 (Fig. 10B). Indeed, this would be consistent with the findings of

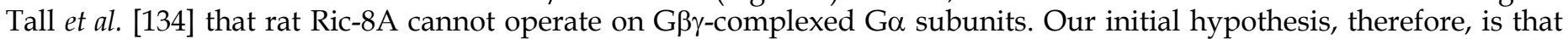
$\mathrm{G} \beta \gamma$ is somehow excluded from or removed from GOA-1/GPA-16 subunits by the GPR-1/-2 GoLoco motif and, perhaps, GPR-1/-2 proteins "present" G $\beta \gamma$-free G $\alpha$ GDP to RIC-8, thus acting cooperatively with RIC-8 to facilitate nucleotide exchange (Fig. 10B). A complication of this theory is that, although GoLoco-derived peptides might be able to 
disrupt a heterotrimer in vitro [115, 124], this "heterotrimer-splitting" activity appears unlikely to happen under physiological conditions (e.g., Fig. 7) [116]. An alternative explanation suggested by the ion channel data of Oxford and Webb is that an initial round of GEF activity is required on the heterotrimer to remove G $\alpha$ GTP from the proximity of G $\beta \gamma$, and then GTP hydrolysis produces G $\alpha$ GDP now competent to interact with the GoLoco motif of GPR-1/2 rather than re-associate with G $\beta \gamma$. RIC-8 cannot provide this initial round of GEF activity if it cannot act on the G $\alpha \beta \gamma$ heterotrimer [134]; thus, either 7TM receptor-mediated GEF activity, another (unknown) GEF activity, or a non-GEF "G $\beta \gamma$ releasing factor" would have to operate on the heterotrimer to establish this pathway.

Another possibility to consider is that the crucial parameter determining the output of the system (i.e., astral MT force generation) is the overall rate of the G $\alpha$ nucleotide cycle, as accelerated by the combined actions of RIC- 8 and RGS7 (Fig. 11). A key observation in this vein is provided by studies from Neubig and colleagues on the effect of RGS proteins on the receptor-catalyzed G-protein cycle. Using reconstituted components, it was observed that, in addition to facilitating steady-state GTP hydrolysis, RGS-box proteins also potently increased receptor-stimulated GTP $\gamma S$ binding [138]. This was not due to a physical scaffolding mechanism, rather a kinetic scaffolding or 'spatial focusing' phenomenon [138]. This has previously been observed in the context of potassium channel regulation in which RGS proteins accelerate the kinetics of both channel activation and deactivation without altering the steady-state level of channel conductance $[139,140]$. Thus in asymmetric cell division, it may be that rapid Ga GTPase cycling promoted by RIC-8 and RGS-7 provides a high-efficiency, spatially-localized signaling complex, sufficient for force generation.

Figure 11. Predicted effects of RIC-8 and RGS-7 on the G $\alpha$ nucleotide cycle underlying MT force generation in ACD. A. Steady-state GTP hydrolysis of $\mathrm{G} \alpha$ subunits is known to be limited by nucleotide exchange $\left(k_{e x}\right)$, not by the intrinsic GTPase rate $\left(k_{c a t}\right)$ [141, 142]. B. RGS-7 GAP activity, while by definition accelerating the intrinsic GTPase rate of $\mathrm{G} \alpha\left(k_{c a t}>k_{\text {cat }}\right)$, will have no effect on steady-state GTP hydrolysis, as the limiting rate for isolated Ga subunits remains GDP release, a key component of $k_{e x}[141,142]$. C. RIC-8 GEF activity should accelerate nucleotide exchange $\left(k_{e x^{\prime}}>k_{e x}\right)$ and increase observed rate of steady-state GTP hydrolysis (up to the new limiting rate of $k_{c a t}$ if $k_{e x^{\prime}}>k_{c a t}$ ). D. Addition of both RIC-8 GEF and RGS-7 GAP activities should dramatically accelerate steady-state GTP hydrolysis $v s$ the basal condition in (A), up to whichever component rate is limiting $\left(k_{e x^{\prime}}\right.$ or $\left.k_{c a t}\right)$. E. A graphical representation of predicted steady state GTP hydrolysis by Ga in the presence of these two novel G-protein cycle components. The ordinate represents the amount of inorganic phosphate (Pi) released, and the abscissa represents time.

\section{4.e. Direct tubulin/G $\alpha$ interaction as a model for the mechanism of MT force generation?}

Forward genetic studies in Drosophila and, especially, C. elegans (Fig. 10A) have pointed to a critical role in metazoans for Gi-family $\mathrm{G} \alpha$ subunits and their regulators in controlling force generation on astral MTs during chromosomal segregation. One simple model to potentially explain this genetic evidence is that $G \alpha_{i}$ subunits interact directly with MTs at their stable tubulin GTP caps to enhance cap stability and MT elongation $[143,144]$, and that this tubulin/ $\mathrm{G} \alpha_{i}$ interaction is dependent on the nucleotide state of $\mathrm{G} \alpha$. Previous findings from Rasenick and colleagues indicate that mammalian $\mathrm{G} \alpha$ subunits directly interact with tubulin and MTs and modulate their dynamics in vitro. High-affinity binding ( $\mathrm{K}_{\mathrm{D}} \approx 120 \mathrm{nM}$; ref. [145]) between $\mathrm{G} \alpha_{\mathrm{i} 1}$ and tubulin GTP has been shown to result in $\mathrm{G \alpha}_{\mathrm{i} 1}$ "transactivation" (i.e., GTP transfer to G $\alpha$ from
$A$

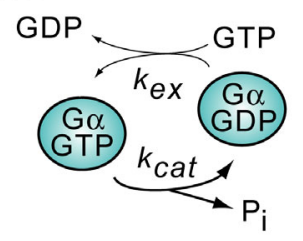

C

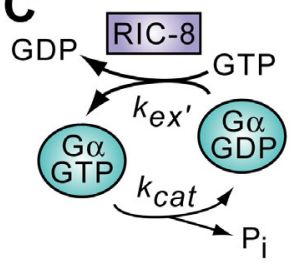

B

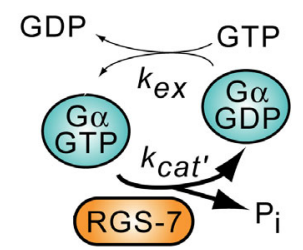

D

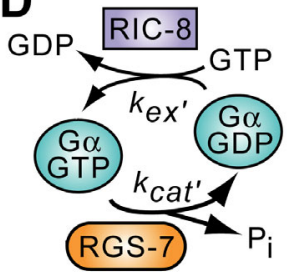

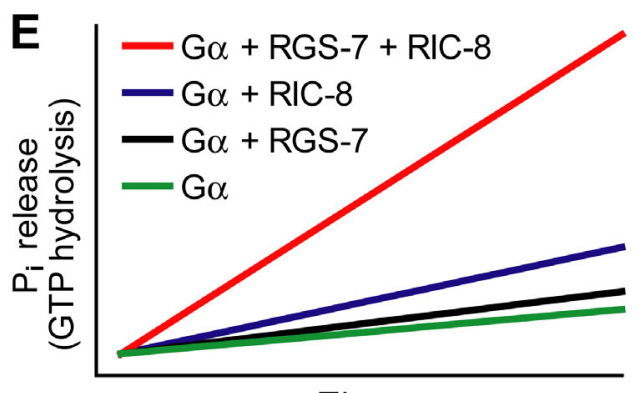

Time the exchangable binding-site on $\beta$-tubulin $[146,147,148,149])$. Moreover, in the absence of GTP or presence of nonhydrolyzable GppNHp, G $\alpha_{i 1}$ serves as a GAP for tubulin GTP in vitro [150], activating its GTPase activity and increasing the frequency of MT "catastrophe" (the transition from slow growth to rapid shortening [151]); these effects of G $\alpha_{i 1}$ are inhibited in the presence of GTP and, indeed, MT assembly by tubulin is enhanced by G $\alpha_{i 1}$ in high GTP levels [150]. Goldstein and colleagues have recently developed a live cell imaging technique, "cortical imaging of microtubule stability" (CIMS), to visualize microtubule dynamics in real-time in the C. elegans zygote [152]. Using CIMS, it was demonstrated that anterior MTs reside longer at the cell cortex than do posterior MTs. However, in goa-1/gpa-16 (RNAi) zygotes, anterior and posterior microtubules have equal stability at the cell cortex. This provides direct evidence that G $\alpha$ subunits regulate microtubule dynamics in vivo and is consistent with a Ga GDP/GPR-1/-2 complex regulating cortical force generation. However, it remains to be directly tested whether direct interactions between tubulin and Ga mediate force generation, or whether members of the kinesin and dynein motor protein superfamilies $[153,154]$ and/or other microtubule-associated proteins at MT ends are the ultimate force generators, with their actions directly modulated by $\mathrm{G} \alpha$ subunits. Future studies in this field should be aimed at delineating the molecular mechanisms by which the novel G $\alpha$ nucleotide cycle, composed of novel GDI, GEF, and GAP activities, communicates with microtubule machinery.

\section{Conclusions}

Since the discovery of the RGS proteins and their Go-directed GAP activity in 1996, there has been a considerable shift in our collective appreciation of RGS protein function in 7TM receptor signal transduction. Far from being mere negative regulators, RGS proteins also serve roles as Ga effectors (e.g., the RGS-RhoGEFs), signaling scaffolds (e.g., 


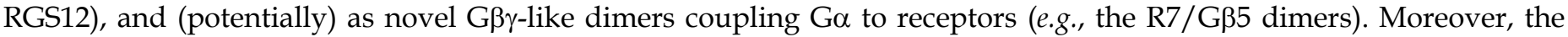
discovery of the GoLoco motif, first found in RGS12 and RGS14, has illuminated a novel GDI activity for Go subunits involved in an equally novel interplay between $G \alpha$ and tubulin controling microtubule force generation critical to chromosomal segregation during cell division. Further exploration of the structural features of RGS protein superfamily members and their functional effects on signal transduction complexes should provide additional surprises.

\section{Conflict of interest}

The authors declare that no conflict of interest exists.

\section{References}

1. Gilman AG. G proteins: transducers of receptor-generated signals. Annu Rev Biochem. 1987 56:615-49.

2. Hamm HE. The many faces of G protein signaling. J Biol Chem. 1998;273(2):669-72.

3. Offermanns S. G-proteins as transducers in transmembrane signalling. Prog Biophys Mol Biol. 2003;83(2):101-30.

4. De Vries L, et al. GAIP, a protein that specifically interacts with the trimeric G protein $\mathrm{G}$ alpha i3, is a member of a protein family with a highly conserved core domain. Proc Natl Acad Sci U S A. 1995;92(25):11916-20.

5. Dohlman HG, et al. Sst2, a negative regulator of pheromone signaling in the yeast Saccharomyces cerevisiae: expression, localization, and genetic interaction and physical association with Gpa1 (the G-protein alpha subunit). Mol Cell Biol. 1996;16(9):5194-209.

6. Druey KM, et al. Inhibition of G-protein-mediated MAP kinase activation by a new mammalian gene family. Nature. 1996;379(6567):742-6.

7. Koelle MR, et al. EGL-10 regulates G protein signaling in the C. elegans nervous system and shares a conserved domain with many mammalian proteins. Cell. 1996;84(1):115-25.

8. Siderovski DP, et al. A new family of regulators of G-protein-coupled receptors? Curr Biol. 1996;6(2):211-2.

9. $\mathrm{Yu} \mathrm{JH}$, et al. The Aspergillus FlbA RGS domain protein antagonizes G protein signaling to block proliferation and allow development. Embo J. 1996;15(19):5184-90.

10. Berman DM, et al. GAIP and RGS4 are GTPase-activating proteins for the Gi subfamily of G protein alpha subunits. Cell. 1996;86(3):445-52.

11. Hunt TW, et al. RGS10 is a selective activator of G alpha i GTPase activity. Nature. 1996;383(6596):175-7.

12. Watson N, et al. RGS family members: GTPase-activating proteins for heterotrimeric G-protein alpha-subunits. Nature. 1996;383(6596):172-5.

13. Siderovski DP, et al. Whither goest the RGS proteins? Crit Rev Biochem Mol Biol. 1999 34(4):215-51.

14. Neubig RR, et al. Regulators of G-protein signalling as new central nervous system drug targets. Nat Rev Drug Discov. 2002;1(3):187-97.

15. Kimple RJ, et al. Established and emerging fluorescence-based assays for G-protein function: heterotrimeric G-protein alpha subunits and regulator of G-protein signaling (RGS) proteins. Comb Chem High Throughput Screen. 2003;6(4):399-407.

16. Arshavsky VY, et al. Lifetime regulation of G protein-effector complex: emerging importance of RGS proteins. Neuron. 1998;20(1):11-4.

17. Brandt DR, et al. GTPase activity of the stimulatory GTP-binding regulatory protein of adenylate cyclase, Gs. Accumulation and turnover of enzyme-nucleotide intermediates. J Biol Chem. 1985;260(1):266-72.

18. Higashijima $\mathrm{T}$, et al. Effects of $\mathrm{Mg} 2+$ and the beta gamma-subunit complex on the interactions of guanine nucleotides with $\mathrm{G}$ proteins. J Biol Chem. 1987;262(2):762-6.

19. Chan RK, et al. Isolation and genetic analysis of Saccharomyces cerevisiae mutants supersensitive to G1 arrest by a factor and alpha factor pheromones. Mol Cell Biol. 1982;2(1):11-20.

20. Chan RK, et al. Physiological characterization of Saccharomyces cerevisiae mutants supersensitive to G1 arrest by a factor and alpha factor pheromones. Mol Cell Biol. 1982;2(1):21-9.

21. von Buchholtz L, et al. RGS21 is a novel regulator of G protein signalling selectively expressed in subpopulations of taste bud cells. Eur J Neurosci. 2004;19(6):1535-44.

22. Wang L, et al. Cloning and mitochondrial localization of full-length D-AKAP2, a protein kinase A anchoring protein. Proc Natl Acad Sci U S A. 2001;98(6):3220-5.

23. Zheng B, et al. Divergence of RGS proteins: evidence for the existence of six mammalian RGS subfamilies. Trends Biochem Sci. 1999;24(11):411-4.

24. Ross EM, et al. GTPase-activating proteins for heterotrimeric G proteins: regulators of $G$ protein signaling (RGS) and RGS-like proteins. Annu Rev Biochem. 2000 69:795-827.

25. Mao H, et al. RGS17/RGSZ2, a novel regulator of Gi/o, Gz, and Gq signaling. J Biol Chem. 2004;279(25):26314-22.

26. Jones TLZ. Role of palmitoylation in RGS protein function. Methods Enzymol. 2004 389:33-55.

27. Snow BE, et al. A G protein gamma subunit-like domain shared between RGS11 and other RGS proteins specifies binding to Gbeta5 subunits. Proc Natl Acad Sci U S A. 1998;95(22):13307-12.

28. Siderovski DP, et al. The GoLoco motif: a Galphai/o binding motif and potential guanine-nucleotide exchange factor. Trends Biochem Sci. 1999;24(9):340-1.

29. Schiff ML, et al. Tyrosine-kinase-dependent recruitment of RGS12 to the N-type calcium channel. Nature. 2000;408(6813):723-7.

30. Spink KE, et al. Structural basis of the Axin-adenomatous polyposis coli interaction. Embo J. 2000;19(10):2270-9.

31. Kikuchi A. Modulation of Wnt signaling by Axin and Axil. Cytokine Growth Factor Rev. 1999;10(3-4):255-65.

32. Zheng B, et al. RGS-PX1, a GAP for GalphaS and sorting nexin in vesicular trafficking. Science. 2001;294(5548):1939-42.

33. Aiyar A. The use of CLUSTAL W and CLUSTAL X for multiple sequence alignment. Methods Mol Biol. 2000 132:221-41.

34. Page RD. TreeView: an application to display phylogenetic trees on personal computers. Comput Appl Biosci. 1996;12(4):357-8.

35. Letunic I, et al. Recent improvements to the SMART domain-based sequence annotation resource. Nucleic Acids Res. 2002;30(1):242-4.

36. Kelley LA, et al. Enhanced genome annotation using structural profiles in the program 3D-PSSM. J Mol Biol. 2000;299(2):499-520.

37. Snow BE, et al. Fidelity of G protein beta-subunit association by the G protein gamma-subunit-like domains of RGS6, RGS7, and RGS11. Proc Natl Acad Sci U S A. 1999;96(11):6489-94.

38. Siderovski DP, et al. Assays of complex formation between RGS protein G gamma subunit-like domains and G beta subunits. Methods Enzymol. $2002344: 702-23$.

39. Makino ER, et al. The GTPase activating factor for transducin in rod photoreceptors is the complex between RGS9 and type 5 G protein beta subunit. Proc Natl Acad Sci U S A. 1999;96(5):1947-52.

40. Levay K, et al. Gbeta5 prevents the RGS7-Galphao interaction through binding to a distinct Ggamma-like domain found in RGS7 and other RGS proteins. Proc Natl Acad Sci U S A. 1999;96(5):2503-7.

41. Witherow DS, et al. Complexes of the G protein subunit gbeta 5 with the regulators of G protein signaling RGS7 and RGS9. Characterization in native tissues and in transfected cells. J Biol Chem. 2000;275(32):24872-80.

42. van der Linden AM, et al. The G-protein beta-subunit GPB-2 in Caenorhabditis elegans regulates the G(o)alpha-G(q)alpha signaling network through interactions with the regulator of G-protein signaling proteins EGL-10 and EAT-16. Genetics. 2001;158(1):221-35.

43. Chase DL, et al. Two RGS proteins that inhibit Galpha(o) and Galpha(q) signaling in C. elegans neurons require a Gbeta(5)-like subunit for function. Curr Biol. 2001;11(4):222-31.

44. Sondek J, et al. Ggamma-like (GGL) domains: new frontiers in G-protein signaling and beta-propeller scaffolding. Biochem Pharmacol. 2001;61(11):1329-37.

45. Jones MB, et al. The Gbeta/gamma dimer as a novel source of selectivity in G-protein signaling: GGL-ing at convention. Mol Interv. 2004;4:20014. 
46. Ponting CP, et al. Pleckstrin's repeat performance: a novel domain in G-protein signaling? Trends Biochem Sci. 1996 Jul;21(7):245-6.

47. Hu G, et al. R9AP, a membrane anchor for the photoreceptor GTPase accelerating protein, RGS9-1. Proc Natl Acad Sci U S A. 2002;99(15):9755-60.

48. Lishko PV, et al. Specific binding of RGS9-Gbeta 5L to protein anchor in photoreceptor membranes greatly enhances its catalytic activity. J Biol Chem. 2002;277(27):24376-81.

49. Hu G, et al. Activation of RGS9-1GTPase acceleration by its membrane anchor, R9AP. J Biol Chem. 2003;278(16):14550-4 .

50. Martemyanov KA, et al. The DEP domain determines subcellular targeting of the GTPase activating protein RGS9 in vivo. J Neurosci. 2003;23(32):10175-81.

51. Nishiguchi KM, et al. Defects in RGS9 or its anchor protein R9AP in patients with slow photoreceptor deactivation. Nature. 2004;427(6969):75-8.

52. Oliveira-Dos-Santos AJ, et al. Regulation of T cell activation, anxiety, and male aggression by RGS2. Proc Natl Acad Sci U S A. 2000;97(22):122727.

53. Tang MK, et al. Regulator of G-protein signaling-2 mediates vascular smooth muscle relaxation and blood pressure. Nat Med. 2003;9(12):1506-12.

54. Heximer SP, et al. Hypertension and prolonged vasoconstrictor signaling in RGS2-deficient mice. J Clin Invest. 2003;111(4):445-52.

55. Grant SL, et al. Specific regulation of RGS2 messenger RNA by angiotensin II in cultured vascular smooth muscle cells. Mol Pharmacol. 2000;57(3):460-7.

56. Snow BE, et al. GTPase activating specificity of RGS12 and binding specificity of an alternatively spliced PDZ (PSD-95/Dlg/ZO-1) domain. J Biol Chem. 1998;273(28):17749-55.

57. Snow BE, et al. Molecular cloning of regulators of G-protein signaling family members and characterization of binding specificity of RGS12 PDZ domain. Methods Enzymol. 2002 344:740-61.

58. Forman-Kay JD, et al. Diversity in protein recognition by PTB domains. Curr Opin Struct Biol. 1999;9(6):690-5.

59. Zeng W, et al. The N-terminal domain of RGS4 confers receptor-selective inhibition of G protein signaling. J Biol Chem. 1998;273(52):34687-90.

60. Xu X, et al. RGS proteins determine signaling specificity of Gq-coupled receptors. J Biol Chem. 1999;274(6):3549-56.

61. Ingi $\mathrm{T}$, et al. Dynamic regulation of RGS2 suggests a novel mechanism in G-protein signaling and neuronal plasticity. J Neurosci. 1998;18(18):7178-88.

62. Wang Q, et al. Receptor-selective effects of endogenous RGS3 and RGS5 regulate mitogen-activated protein kinase activation in rat vascular smooth muscle cells. J Biol Chem. 2002;277(28):24949-58.

63. Bernstein LS, et al. RGS2 binds directly and selectively to the M1 muscarinic acetylcholine receptor third intracellular loop to modulate Gq/11alpha signaling. J Biol Chem. 2004;279(20):21248-56.

64. Chen JG, et al. A seven-transmembrane RGS protein that modulates plant cell proliferation. Science. 2003;301(5640):1728-31.

65. Willard FS, et al. Purification and in vitro functional analysis of the Arabidopsis thaliana Regulator of G-Protein Signaling-1. Methods Enzymol. 2004 389:320-37.

66. Chen J-G, et al. AtRGS1 function in Arabidopsis thaliana. Methods Enzymol. 2004 389:338-50.

67. Hepler JR, et al. RGS4 and GAIP are GTPase-activating proteins for Gq alpha and block activation of phospholipase C beta by gamma-thio-GTPGq alpha. Proc Natl Acad Sci U S A. 1997;94(2):428-32.

68. Carman CV, et al. Selective regulation of Galpha(q/11) by an RGS domain in the G protein-coupled receptor kinase, GRK2. J Biol Chem. 1999;274(48):34483-92.

69. Sallese $\mathrm{M}$, et al. Selective regulation of $\mathrm{Gq}$ signaling by $\mathrm{G}$ protein-coupled receptor kinase 2: direct interaction of kinase $\mathrm{N}$ terminus with activated galphaq. Mol Pharmacol. 2000;57(4):826-31.

70. Anger T, et al. Differential contribution of GTPase activation and effector antagonism to the inhibitory effect of RGS proteins on Gq-mediated signaling in vivo. J Biol Chem. 2004;279(6):3906-15.

71. Kaibuchi K, et al. Regulation of the cytoskeleton and cell adhesion by the Rho family GTPases in mammalian cells. Annu Rev Biochem. 1999 68:459-86.

72. Sah VP, et al. The role of Rho in G protein-coupled receptor signal transduction. Annu Rev Pharmacol Toxicol. 2000 40:459-89.

73. Snyder JT, et al. Structural basis for the selective activation of Rho GTPases by Dbl exchange factors. Nat Struct Biol. 2002;9(6):468-75.

74. Kozasa T, et al. p115 RhoGEF, a GTPase activating protein for Galpha12 and Galpha13. Science. 1998;280(5372):2109-11.

75. Hart MJ, et al. Direct stimulation of the guanine nucleotide exchange activity of p115 RhoGEF by Galpha13. Science. 1998;280(5372):2112-4.

76. Booden MA, et al. Leukemia-associated Rho guanine nucleotide exchange factor promotes $\mathrm{G}$ alpha q-coupled activation of RhoA. Mol Cell Biol. 2002;22(12):4053-61.

77. Vogt S, et al. Receptor-dependent RhoA activation in G12/G13-deficient cells: genetic evidence for an involvement of Gq/G11. J Biol Chem. 2003;278(31):28743-9.

78. Suzuki N, et al. Galpha 12 activates Rho GTPase through tyrosine-phosphorylated leukemia-associated RhoGEF. Proc Natl Acad Sci U S A. 2003;100(2):733-8.

79. Chikumi H, et al. Regulation of G protein-linked guanine nucleotide exchange factors for Rho, PDZ-RhoGEF, and LARG by tyrosine phosphorylation: evidence of a role for focal adhesion kinase. J Biol Chem. 2002;277(14):12463-73.

80. Wang Q, et al. Thrombin and Lysophosphatidic Acid Receptors Utilize Distinct rhoGEFs in Prostate Cancer Cells. J Biol Chem. 2004;279(28):28831-4.

81. Taya S, et al. Direct interaction of insulin-like growth factor-1 receptor with leukemia-associated RhoGEF. J Cell Biol. 2001;155(5):809-20.

82. Becknell $\mathrm{B}$, et al. Characterization of leukemia-associated Rho guanine nucleotide exchange factor (LARG) expression during murine development. Cell Tissue Res. 2003;314(3):361-6.

83. Kumanogoh A, et al. Biological functions and signaling of a transmembrane semaphorin, CD100/Sema4D. Cell Mol Life Sci. 2004;61(3):292-300.

84. Aurandt J, et al. The semaphorin receptor plexin-B1 signals through a direct interaction with the Rho-specific nucleotide exchange factor, LARG. Proc Natl Acad Sci U S A. 2002;99(19):12085-90.

85. Driessens $\mathrm{MH}$, et al. B plexins activate Rho through PDZ-RhoGEF. FEBS Lett. 2002;529(2-3):168-72.

86. Hirotani $\mathrm{M}$, et al. Interaction of plexin-B1 with PDZ domain-containing Rho guanine nucleotide exchange factors. Biochem Biophys Res Commun. 2002;297(1):32-7.

87. Perrot V, et al. Plexin B regulates Rho through the guanine nucleotide exchange factors leukemia-associated Rho GEF (LARG) and PDZ-RhoGEF. J Biol Chem. 2002;277(45):43115-20.

88. Swiercz JM, et al. Plexin-B1 directly interacts with PDZ-RhoGEF/LARG to regulate RhoA and growth cone morphology. Neuron. 2002;35(1):5163.

89. Hunt RA, et al. Snapin interacts with the N-terminus of regulator of G protein signaling 7. Biochem Biophys Res Commun. 2003;303(2):594-9.

90. Takesono A, et al. Receptor-independent activators of heterotrimeric G-protein signaling pathways. J Biol Chem. 1999;274(47):33202-5.

91. Willard FS, et al. Return of the GDI: The GoLoco motif in cell division. Annu Rev Biochem. 2004 73:925-51.

92. Kimple RJ, et al. Structural determinants for GoLoco-induced inhibition of nucleotide release by Galpha subunits. Nature. 2002;416(6883):878-81.

93. Peterson YK, et al. Stabilization of the GDP-bound conformation of Gialpha by a peptide derived from the G-protein regulatory motif of AGS3. J Biol Chem. 2000;275(43):33193-6.

94. Kimple RJ, et al. Guanine nucleotide dissociation inhibitor activity of the triple GoLoco motif protein G18: alanine-to-aspartate mutation restores function to an inactive second GoLoco motif. Biochem J. 2004;378(Pt 3):801-8.

95. Snow BE, et al. Molecular cloning and expression analysis of rat Rgs12 and Rgs14. Biochem Biophys Res Commun. 1997;233(3):770-7.

96. Kimple RJ, et al. RGS12 and RGS14 GoLoco motifs are G alpha(i) interaction sites with guanine nucleotide dissociation inhibitor Activity. J Biol Chem. 2001;276(31):29275-81. 
97. Hollinger S, et al. RGS14 is a bifunctional regulator of Galphai/o activity that exists in multiple populations in brain. J Neurochem. 2001;79(5):941-9.

98. Traver S, et al. RGS14 is a novel Rap effector that preferentially regulates the GTPase activity of galphao. Biochem J. 2000;350(Pt 1):19-29.

99. Mochizuki N, et al. Identification and cDNA cloning of a novel human mosaic protein, LGN, based on interaction with G alpha i2. Gene. 1996;181(1-2):39-43.

100. Schaefer M, et al. A protein complex containing Inscuteable and the Galpha-binding protein Pins orients asymmetric cell divisions in Drosophila. Curr Biol. 2000;10(7):353-62.

101. Yu F, et al. Analysis of partner of inscuteable, a novel player of Drosophila asymmetric divisions, reveals two distinct steps in inscuteable apical localization. Cell. 2000;100(4):399-409.

102. Colombo K, et al. Translation of polarity cues into asymmetric spindle positioning in Caenorhabditis elegans embryos. Science. 2003;300(5627):1957-61.

103. Srinivasan DG, et al. A complex of LIN-5 and GPR proteins regulates G protein signaling and spindle function in C elegans. Genes Dev. 2003;17(10):1225-39.

104. Gotta M, et al. Asymmetrically distributed C. elegans homologs of AGS3/PINS control spindle position in the early embryo. Curr Biol. 2003;13(12):1029-37.

105. Oberdick J, et al. A Purkinje cell differentiation marker shows a partial DNA sequence homology to the cellular sis/PDGF2 gene. Neuron. 1988;1(5):367-76.

106. Mochizuki N, et al. Activation of the ERK/MAPK pathway by an isoform of rap1GAP associated with G alpha(i). Nature. 1999;400(6747):891-4.

107. Natochin M, et al. Inhibition of GDP/GTP exchange on G alpha subunits by proteins containing G-protein regulatory motifs. Biochemistry. 2001;40(17):5322-8.

108. Meng J, et al. Functional interaction between Galpha(z) and Rap1GAP suggests a novel form of cellular cross-talk. J Biol Chem. 1999;274(51):36663-9.

109. Meng J, et al. Activation of Gz attenuates Rap1-mediated differentiation of PC12 cells. J Biol Chem. 2002;277(45):43417-24.

110. Jordan JD, et al. Modulation of rap activity by direct interaction of Galpha(o) with Rap1 GTPase-activating protein. J Biol Chem. 1999;274(31):21507-10.

111. Ma H, et al. Influence of cytosolic AGS3 on receptor--G protein coupling. Biochemistry. 2003;42(26):8085-93.

112. Sato M, et al. AGS3 and signal integration by Galpha(s)- and Galpha(i)-coupled receptors: AGS3 blocks the sensitization of adenylyl cyclase following prolonged stimulation of a Galpha(i)-coupled receptor by influencing processing of Galpha(i). J Biol Chem. 2004;279(14):13375-82.

113. Bowers MS, et al. AGS3: a G-Protein regulator of addiction-associated behaviors. Ann N Y Acad Sci. 2003;1003:356-7.

114. Kimple RJ, et al. The GoLoco motif: heralding a new tango between G protein signaling and cell division. Mol Interv. 2002;2(2):88-100.

115. Ghosh M, et al. Receptor- and nucleotide exchange-independent mechanisms for promoting G protein subunit dissociation. J Biol Chem. 2003;278(37):34747-50.

116. Oxford GS, et al. GoLoco motif peptides as probes of G-alpha subunit specificity in coupling of GPCRs to ion channels. Methods Enzymol. 2004 390:437-50.

117. Knoblich JA. Asymmetric cell division during animal development. Nat Rev Mol Cell Biol. 2001;2(1):11-20.

118. Schober M, et al. Bazooka recruits Inscuteable to orient asymmetric cell divisions in Drosophila neuroblasts. Nature. 1999;402(6761):548-51.

119. Wodarz A, et al. Bazooka provides an apical cue for Inscuteable localization in Drosophila neuroblasts. Nature. 1999;402(6761):544-7.

120. Kraut R, et al. Role of inscuteable in orienting asymmetric cell divisions in Drosophila. Nature. 1996;383(6595):50-5.

121. Knoblich JA, et al. Deletion analysis of the Drosophila Inscuteable protein reveals domains for cortical localization and asymmetric localization. Curr Biol. 1999;9(3):155-8.

122. Tio M, et al. A functional analysis of inscuteable and its roles during Drosophila asymmetric cell divisions. J Cell Sci. $1999 ; 112$ (Pt 10):1541-51.

123. Parmentier ML, et al. Rapsynoid/partner of inscuteable controls asymmetric division of larval neuroblasts in Drosophila. J Neurosci. 2000;20(14):RC84.

124. Schaefer M, et al. Heterotrimeric G proteins direct two modes of asymmetric cell division in the Drosophila nervous system. Cell. 2001;107(2):18394.

125. Fuse N, et al. Heterotrimeric G proteins regulate daughter cell size asymmetry in Drosophila neuroblast divisions. Curr Biol. 2003;13(11):947-54.

126. Macara IG. Parsing the polarity code. Nat Rev Mol Cell Biol. 2004;5(3):220-31.

127. Gotta M, et al. Distinct roles for Galpha and Gbetagamma in regulating spindle position and orientation in Caenorhabditis elegans embryos. Nat Cell Biol. 2001;3(3):297-300.

128. Grill SW, et al. The distribution of active force generators controls mitotic spindle position. Science. 2003;301(5632):518-21.

129. Gonczy P, et al. Functional genomic analysis of cell division in C. elegans using RNAi of genes on chromosome III. Nature. 2000;408(6810):331-6.

130. Blatch GL, et al. The tetratricopeptide repeat: a structural motif mediating protein-protein interactions. Bioessays. 1999;21(11):932-9.

131. Miller KG, et al. A genetic selection for Caenorhabditis elegans synaptic transmission mutants. Proc Natl Acad Sci U S A. 1996;93(22):12593-8.

132. Miller KG, et al. RIC-8 (Synembryn): a novel conserved protein that is required for G(q)alpha signaling in the C. elegans nervous system. Neuron. 2000;27(2):289-99.

133. Miller KG, et al. A role for RIC-8 (Synembryn) and GOA-1 (G(o)alpha) in regulating a subset of centrosome movements during early embryogenesis in Caenorhabditis elegans. Genetics. 2000;156(4):1649-60.

134. Tall GG, et al. Mammalian Ric-8A (synembryn) is a heterotrimeric Galpha protein guanine nucleotide exchange factor. J Biol Chem. 2003;278(10):8356-62.

135. Ferguson KM, et al. The influence of bound GDP on the kinetics of guanine nucleotide binding to G proteins. J Biol Chem. 1986;261(16):7393-9.

136. Plusa B, et al. Site of the previous meiotic division defines cleavage orientation in the mouse embryo. Nat Cell Biol. 2002;4(10):811-5.

137. Cayouette M, et al. Asymmetric segregation of Numb in retinal development and the influence of the pigmented epithelium. J Neurosci. 2001;21(15):5643-51.

138. Zhong H, et al. A spatial focusing model for $G$ protein signals. Regulator of $G$ protein signaling (RGS) protien-mediated kinetic scaffolding. J Biol Chem. 2003;278(9):7278-84.

139. Saitoh O, et al. RGS8 accelerates G-protein-mediated modulation of K+ currents. Nature. 1997;390(6659):525-9.

140. Doupnik CA, et al. RGS proteins reconstitute the rapid gating kinetics of gbetagamma-activated inwardly rectifying K+ channels. Proc Natl Acad Sci U S A. 1997;94(19):10461-6.

141. Mukhopadhyay S, et al. Quench-flow kinetic measurement of individual reactions of G-protein-catalyzed GTPase cycle. Methods Enzymol. 2002 344:350-69.

142. McEwen DP, et al. Fluorescence approaches to study G protein mechanisms. Methods Enzymol. 2002 344:403-20.

143. Carlier MF. Role of nucleotide hydrolysis in the dynamics of actin filaments and microtubules. Int Rev Cytol. 1989 115:139-70.

144. Erickson HP, et al. Microtubule dynamic instability and GTP hydrolysis. Annu Rev Biophys Biomol Struct. 1992 21:145-66.

145. Wang N, et al. Tubulin binds specifically to the signal-transducing proteins, Gs alpha and Gi alpha 1. J Biol Chem. 1990;265(3):1239-42.

146. Rasenick MM, et al. Exchange of guanine nucleotides between tubulin and GTP-binding proteins that regulate adenylate cyclase: cytoskeletal modification of neuronal signal transduction. J Neurochem. 1988;51(1):300-11.

147. Roychowdhury S, et al. G protein binding and G protein activation by nucleotide transfer involve distinct domains on tubulin: regulation of signal transduction by cytoskeletal elements. Biochemistry. 1993;32(18):4955-61.

148. Yan K, et al. Tubulin stimulates adenylyl cyclase activity in C6 glioma cells by bypassing the beta-adrenergic receptor: a potential mechanism of G protein activation. J Neurochem. 2001;76(1):182-90. 
149. Chen NF, et al. A specific domain of Gialpha required for the transactivation of Gialpha by tubulin is implicated in the organization of cellular microtubules. J Biol Chem. 2003;278(17):15285-90.

150. Roychowdhury S, et al. G protein alpha subunits activate tubulin GTPase and modulate microtubule polymerization dynamics. J Biol Chem. 1999;274(19):13485-90.

151. Walker RA, et al. Dynamic instability of individual microtubules analyzed by video light microscopy: rate constants and transition frequencies. J Cell Biol. 1988;107(4):1437-48.

152. Labbe JC, et al. PAR proteins regulate microtubule dynamics at the cell cortex in C. elegans. Curr Biol. 2003;13(9):707-14.

153. Mountain V, et al. Dissecting the role of molecular motors in the mitotic spindle. Anat Rec. 2000;261(1):14-24.

154. Hunter AW, et al. How motor proteins influence microtubule polymerization dynamics. J Cell Sci. 2000;113(Pt 24):4379-89. 